\title{
Properties and Applications of Programs with Monotone and Convex Constraints
}

\author{
Lengning Liu \\ Mirosław Truszczyński \\ Department of Computer Science, University of Kentucky, \\ Lexington, KY 40506-0046, USA
}

LLIU1@CS.UKY.EDU

\begin{abstract}
We study properties of programs with monotone and convex constraints. We extend to these formalisms concepts and results from normal logic programming. They include the notions of strong and uniform equivalence with their characterizations, tight programs and Fages Lemma, program completion and loop formulas. Our results provide an abstract account of properties of some recent extensions of logic programming with aggregates, especially the formalism of lparse programs. They imply a method to compute stable models of lparse programs by means of off-theshelf solvers of pseudo-boolean constraints, which is often much faster than the smodels system.
\end{abstract}

\section{Introduction}

We study programs with monotone constraints (Marek \& Truszczyński, 2004; Marek, Niemelä, \& Truszczyński, 2004, 2006) and introduce a related class of programs with convex constraints. These formalisms allow constraints to appear in the heads of program rules, which sets them apart from other recent proposals for integrating constraints into logic programs (Pelov, 2004; Pelov, Denecker, \& Bruynooghe, 2004, 2006; Dell'Armi, Faber, Ielpa, Leone, \& Pfeifer, 2003; Faber, Leone, \& Pfeifer, 2004), and makes them suitable as an abstract basis for formalisms such as lparse programs (Simons, Niemelä, \& Soininen, 2002).

We show that several results from normal logic programming generalize to programs with monotone constraints. We also discuss how these techniques and results can be extended further to the setting of programs with convex constraints. We then apply some of our general results to design and implement a method to compute stable models of lparse programs and show that it is often much more effective than smodels (Simons et al., 2002).

Normal logic programming with the semantics of stable models is an effective knowledge representation formalism, mostly due to its ability to express default assumptions (Baral, 2003; Gelfond \& Leone, 2002). However, modeling numeric constraints on sets in normal logic programming is cumbersome, requires auxiliary atoms and leads to large programs hard to process efficiently. Since such constraints, often called aggregates, are ubiquitous, researchers proposed extensions of normal logic programming with explicit means to express aggregates, and generalized the stable-model semantics to the extended settings.

Aggregates imposing bounds on weights of sets of atoms and literals, called weight constraints, are especially common in practical applications and are included in all recent extensions of logic programs with aggregates. Typically, these extensions do not allow aggregates to appear in the 
heads of rules. A notable exception is the formalism of programs with weight constraints (Niemelä, Simons, \& Soininen, 1999; Simons et al., 2002), which we refer to as lparse programs ${ }^{1}$.

Lparse programs are logic programs whose rules have weight constraints in their heads and whose bodies are conjunctions of weight constraints. Normal logic programs can be viewed as a subclass of lparse programs and the semantics of lparse programs generalizes the stable-model semantics of normal logic programs (Gelfond \& Lifschitz, 1988). Lparse programs are one of the most commonly used extensions of logic programming with weight constraints.

Since rules in lparse programs may have weight constraints as their heads, the concept of onestep provability is nondeterministic, which hides direct parallels between lparse and normal logic programs. An explicit connection emerged when Marek and Truszczyński (2004) and Marek et al. (2004, 2006) introduced logic programs with monotone constraints. These programs allow aggregates in the heads of rules and support nondeterministic computations. Marek and Truszczyński (2004) and Marek et al. (2004, 2006) proposed a generalization of the van Emden-Kowalski onestep provability operator to account for that nondeterminism, defined supported and stable models for programs with monotone constraints that mirror their normal logic programming counterparts, and showed encodings of smodels programs as programs with monotone constraints.

In this paper, we continue investigations of programs with monotone constraints. We show that the notions of uniform and strong equivalence of programs (Lifschitz, Pearce, \& Valverde, 2001; Lin, 2002; Turner, 2003; Eiter \& Fink, 2003) extend to programs with monotone constraints, and that their characterizations (Turner, 2003; Eiter \& Fink, 2003) generalize, too.

We adapt to programs with monotone constraints the notion of a tight program (Erdem \& Lifschitz, 2003) and generalize Fages Lemma (Fages, 1994).

We introduce extensions of propositional logic with monotone constraints. We define the completion of a monotone-constraint program with respect to this logic, and generalize the notion of a loop formula. We then prove the loop-formula characterization of stable models of programs with monotone constraints, extending to the setting of monotone-constraint programs results obtained for normal logic programs by Clark (1978) and Lin and Zhao (2002).

Programs with monotone constraints make explicit references to the default negation operator. We show that by allowing a more general class of constraints, called convex, default negation can be eliminated from the language. We argue that all results in our paper extend to programs with convex constraints.

Our paper shows that programs with monotone and convex constraints have a rich theory that closely follows that of normal logic programming. It implies that programs with monotone and convex constraints form an abstract generalization of extensions of normal logic programs. In particular, all results we obtain in the abstract setting of programs with monotone and convex constraints specialize to lparse programs and, in most cases, yield results that are new.

These results have practical implications. The properties of the program completion and loop formulas, when specialized to the class of lparse programs, yield a method to compute stable models of lparse programs by means of solvers of pseudo-boolean constraints, developed by the propositional satisfiability and integer programming communities (Eén \& Sörensson, 2003; Aloul, Ramani, Markov, \& Sakallah, 2002; Walser, 1997; Manquinho \& Roussel, 2005; Liu \& Truszczyński, 2003). We describe this method in detail and present experimental results on its performance. The results show that our method on problems we used for testing typically outperforms smodels.

1. Aggregates in the heads of rules have also been studied recently by Son and Pontelli (2006) and Son, Pontelli, and Tu (2006). 


\section{Preliminaries}

We consider the propositional case only and assume a fixed set $A t$ of propositional atoms. It does not lead to loss of generality, as it is common to interpret programs with variables in terms of their propositional groundings.

The definitions and results we present in this section come from papers by Marek and Truszczyński (2004) and Marek et al. (2006). Some of them are more general as we allow constraints with infinite domains and programs with inconsistent constraints in the heads.

Constraints. A constraint is an expression $A=(X, C)$, where $X \subseteq A t$ and $C \subseteq \mathcal{P}(X)(\mathcal{P}(X)$ denotes the powerset of $X)$. We call the set $X$ the domain of the constraint $A=(X, C)$ and denote it by $\operatorname{Dom}(A)$. Informally speaking, a constraint $(X, C)$ describes a property of subsets of its domain, with $C$ consisting precisely of these subsets of $X$ that satisfy the constraint (have property) $C$.

In the paper, we identify truth assignments (interpretations) with the sets of atoms they assign the truth value true. That is, given an interpretation $M \subseteq A t$, we have $M \models a$ if and only if $a \in M$. We say that an interpretation $M \subseteq$ At satisfies a constraint $A=(X, C)(M \models A)$, if $M \cap X \in C$. Otherwise, $M$ does not satisfy $A,(M \not \forall A)$.

A constraint $A=(X, C)$ is consistent if there is $M$ such that $M \models A$. Clearly, a constraint $A=(X, C)$ is consistent if and only if $C \neq \emptyset$.

We note that propositional atoms can be regarded as constraints. Let $a \in A t$ and $M \subseteq A t$. We define $C(a)=(\{a\},\{\{a\}\})$. It is evident that $M \models C(a)$ if and only if $M \models a$. Therefore, in the paper we often write $a$ as a shorthand for the constraint $C(a)$.

Constraint programs. Constraints are building blocks of rules and programs. Marek and Truszczyński (2004) defined constraint programs as sets of constraint rules

$$
A \leftarrow A_{1}, \ldots, A_{k}, \operatorname{not}\left(A_{k+1}\right), \ldots, \operatorname{not}\left(A_{m}\right)
$$

where $A, A_{1}, \ldots, A_{n}$ are constraints and not is the default negation operator.

In the context of constraint programs, we refer to constraints and negated constraints as literals. Given a rule $r$ of the form (1), the constraint (literal) $A$ is the head of $r$ and the set $\left\{A_{1}, \ldots\right.$, $\left.A_{k}, \ldots, \operatorname{not}\left(A_{k+1}\right), \ldots, \operatorname{not}\left(A_{m}\right)\right\}$ of literals is the body of $r^{2}$. We denote the head and the body of $r$ by $h d(r)$ and $b d(r)$, respectively. We define the the headset of $r$, written $h s e t(r)$, as the domain of the head of $r$. That is, $h \operatorname{set}(r)=\operatorname{Dom}(h d(r))$.

For a constraint program $P$, we denote by $A t(P)$ the set of atoms that appear in the domains of constraints in $P$. We define the headset of $P$, written $h$ set $(P)$, as the union of the headsets of all rules in $P$.

Models. The concept of satisfiability extends in a standard way to literals $\operatorname{not}(A)(M \models \operatorname{not}(A)$ if $M \not \models A$ ), to sets (conjunctions) of literals and, finally, to constraint programs.

$M$-applicable rules. Let $M \subseteq A t$ be an interpretation. A rule (1) is $M$-applicable if $M$ satisfies every literal in $b d(r)$. We denote by $P(M)$ the set of all $M$-applicable rules in $P$.

Supported models. Supportedness is a property of models. Intuitively, every atom $a$ in a supported model must have "reasons" for being "in". Such reasons are $M$-applicable rules whose heads contain $a$ in their domains. Formally, let $P$ be a constraint program and $M$ a subset of $A t(P)$. A model $M$ of $P$ is supported if $M \subseteq h \operatorname{set}(P(M))$.

Examples. We illustrate the concept with examples. Let $P$ be the constraint program that consists of the following two rules:

2. Sometimes we view the body of a rule as the conjunction of its literals. 


$$
\begin{aligned}
& (\{c, d, e\},\{\{c\},\{d\},\{e\},\{c, d, e\}\}) \leftarrow \\
& (\{a, b\},\{\{a\},\{b\}\}) \leftarrow(\{c, d\},\{\{c\},\{c, d\}\}), \operatorname{not}((\{e\},\{\{e\}\}))
\end{aligned}
$$

A set $M=\{a, c\}$ is a model of $P$ as $M$ satisfies the heads of the two rules. Both rules in $P$ are $M$-applicable. The first of them provides the support for $c$, the second one - for $a$. Thus, $M$ is a supported model.

A set $M^{\prime}=\{a, c, d, e\}$ is also a model of $P$. However, $a$ has no support in $P$. Indeed, $a$ only appears in the headset of the second rule. This rule is not $M^{\prime}$-applicable and so, it does not support $a$. Therefore, $M^{\prime}$ is not a supported model of $P$.

Nondeterministic one-step provability. Let $P$ be a constraint program and $M$ a set of atoms. A set $M^{\prime}$ is nondeterministically one-step provable from $M$ by means of $P$, if $M^{\prime} \subseteq h \operatorname{set}(P(M))$ and $M^{\prime} \models h d(r)$, for every rule $r$ in $P(M)$.

The nondeterministic one-step provability operator $T_{P}^{n d}$ for a program $P$ is an operator on $\mathcal{P}(A t)$ such that for every $M \subseteq A t, T_{P}^{n d}(M)$ consists of all sets that are nondeterministically one-step provable from $M$ by means of $P$.

The operator $T_{P}^{n d}$ is nondeterministic as it assigns to each $M \subseteq A t$ a family of subsets of At, each being a possible outcome of applying $P$ to $M$. In general, $T_{P}^{n d}$ is partial, since there may be sets $M$ such that $T_{P}^{n d}(M)=\emptyset$ (no set can be derived from $M$ by means of $P$ ). For instance, if $P(M)$ contains a rule $r$ such that $h d(r)$ is inconsistent, then $T_{P}^{n d}(M)=\emptyset$.

Monotone constraints. A constraint $(X, C)$ is monotone if $C$ is closed under superset, that is, for every $W, Y \subseteq X$, if $W \in C$ and $W \subseteq Y$ then $Y \in C$.

Cardinality and weight constraints provide examples of monotone constraints. Let $X$ be a finite set and let $C_{k}(X)=\{Y: Y \subseteq X, k \leq|Y|\}$, where $k$ is a non-negative integer. Then $\left(X, C_{k}(X)\right)$ is a constraint expressing the property that a subset of $X$ has at least $k$ elements. We call it a lowerbound cardinality constraint on $X$ and denote it by $k X$.

A more general class of constraints are weight constraints. Let $X$ be a finite set, say $X=$ $\left\{x_{1}, \ldots, x_{n}\right\}$, and let $w, w_{1}, \ldots, w_{n}$ be non-negative reals. We interpret each $w_{i}$ as the weight assigned to $x_{i}$. A lower-bound weight constraint is a constraint of the form $\left(X, C_{w}\right)$, where $C_{w}$ consists of those subsets of $X$ whose total weight (the sum of weights of elements in the subset) is at least $w$. We write it as

$$
w\left[x_{1}=w_{1}, \ldots, x_{n}=w_{n}\right]
$$

If all weights are equal to 1 and $w$ is an integer, weight constraints become cardinality constraints. We also note that the constraint $C(a)$ is a cardinality constraint $1\{a\}$ and also a weight constraint $1[a=1]$. Finally, we observe that lower-bound cardinality and weight constraints are monotone.

Cardinality and weight constraints (in a somewhat more general form) appear in the language of lparse programs (Simons et al., 2002), which we discuss later in the paper. The notation we adopted for these constraints in this paper follows the one proposed by Simons et al. (2002).

We use cardinality and weight constraints in some of our examples. They are also the focus of the last part of the paper, where we use our abstract results to design a new algorithm to compute models of lparse programs.

Monotone-constraint programs. We call constraint programs built of monotone constraints monotone-constraint programs or programs with monotone constraints. That is, monotone-constraint programs consist of rules of rules of the form (1), where $A, A_{1}, \ldots, A_{m}$ are monotone constraints. 
From now on, unless explicitly stated otherwise, programs we consider are monotone-constraint programs.

\subsection{Horn Programs and Bottom-up Computations}

Since we allow constraints with infinite domains and inconsistent constraints in heads of rules, the results given in this subsection are more general than their counterparts by Marek and Truszczyński (2004) and Marek et al. (2004, 2006). Thus, for the sake of completeness, we present them with proofs.

A rule (1) is Horn if $k=m$ (no occurrences of the negation operator in the body or, equivalently, only monotone constraints). A constraint program is Horn if every rule in the program is Horn.

With a Horn constraint program we associate bottom-up computations, generalizing the corresponding notion of a bottom-up computation for a normal Horn program.

Definition 1. Let $P$ be a Horn program. A $P$-computation is a (transfinite) sequence $\left\langle X_{\alpha}\right\rangle$ such that

1. $X_{0}=\emptyset$,

2. for every ordinal number $\alpha, X_{\alpha} \subseteq X_{\alpha+1}$ and $X_{\alpha+1} \in T_{P}^{n d}\left(X_{\alpha}\right)$,

3. for every limit ordinal $\alpha, X_{\alpha}=\bigcup_{\beta<\alpha} X_{\beta}$.

Let $t=\left\langle X_{\alpha}\right\rangle$ be a $P$-computation. Since for every $\beta<\beta^{\prime}, X_{\beta} \subseteq X_{\beta^{\prime}} \subseteq A t$, there is a least ordinal number $\alpha_{t}$ such that $X_{\alpha_{t}+1}=X_{\alpha_{t}}$, in other words, a least ordinal when the $P$-computation stabilizes. We refer to $\alpha_{t}$ as the length of the $P$-computation $t$.

Examples. Here is a simple example showing that some programs have computations of length exceeding $\omega$ and so, the transfinite induction in the definition cannot be avoided. Let $P$ be the program consisting of the following rules:

$$
\begin{aligned}
& \left(\left\{a_{0}\right\},\left\{\left\{a_{0}\right\}\right\}\right) \leftarrow . \\
& \left(\left\{a_{i}\right\},\left\{\left\{a_{i}\right\}\right\}\right) \leftarrow\left(X_{i-1},\left\{X_{i-1}\right\}\right), \text { for } i=1,2, \ldots \\
& (\{a\},\{\{a\}\}) \leftarrow\left(X_{\infty},\left\{X_{\infty}\right\}\right),
\end{aligned}
$$

where $X_{i}=\left\{a_{0}, \ldots a_{i}\right\}, 0 \leq i$, and $X_{\infty}=\left\{a_{0}, a_{1}, \ldots\right\}$. Since the body of the last rule contains a constraint with an infinite domain $X_{\infty}$, it does not become applicable in any finite step of computation. However, it does become applicable in the step $\omega$ and so, $a \in X_{\omega+1}$. Consequently, $X_{\omega+1} \neq X_{\omega}$.

For a $P$-computation $t=\left\langle X_{\alpha}\right\rangle$, we call $\bigcup_{\alpha} X_{\alpha}$ the result of the computation and denote it by $R_{t}$. Directly from the definitions, it follows that $R_{t}=X_{\alpha_{t}}$.

Proposition 1. Let $P$ be a Horn constraint program and t a $P$-computation. Then $R_{t}$ is a supported model of $P$.

Proof. Let $M=R_{t}$ be the result of a $P$-computation $t=\left\langle X_{\alpha}\right\rangle$. We need to show that: (1) $M$ is a model of $P$; and (2) $M \subseteq$ hset $(P(M))$.

(1) Let us consider a rule $r \in P$ such that $M \models b d(r)$. Since $M=R_{t}=X_{\alpha_{t}}$ (where $\alpha_{t}$ is the length of $t$ ), $X_{\alpha_{t}} \models b d(r)$. Thus, $X_{\alpha_{t}+1} \models h d(r)$. Since $M=X_{\alpha_{t}+1}, M$ is a model of $r$ and, consequently, of $P$, as well. 
(2) We will prove by induction that, for every set $X_{\alpha}$ in the computation $t, X_{\alpha} \subseteq h \operatorname{set}(P(M))$. The base case holds since $X_{0}=\emptyset \subseteq h \operatorname{set}(P(M))$.

If $\alpha=\beta+1$, then $X_{\alpha} \in T_{P}^{n d}\left(X_{\beta}\right)$. It follows that $X_{\alpha} \subseteq$ hset $\left(P\left(X_{\beta}\right)\right)$. Since $P$ is a Horn program and $X_{\beta} \subseteq M$, hset $\left(P\left(X_{\beta}\right)\right) \subseteq$ hset $(P(M))$. Therefore, $X_{\alpha} \subseteq$ hset $(P(M))$.

If $\alpha$ is a limit ordinal, then $X_{\alpha}=\bigcup_{\beta<\alpha} X_{\beta}$. By the induction hypothesis, for every $\beta<\alpha$, $X_{\beta} \subseteq h \operatorname{set}(P(M))$. Thus, $X_{\alpha} \subseteq$ hset $(P(M))$. By induction, $M \subseteq h \operatorname{set}(P(M))$.

Derivable models. We use computations to define derivable models of Horn constraint programs. A set $M$ of atoms is a derivable model of a Horn constraint program $P$ if for some $P$-computation $t$, we have $M=R_{t}$. By Proposition 1, derivable models of $P$ are supported models of $P$ and so, also models of $P$.

Derivable models are similar to the least model of a normal Horn program in that both can be derived from a program by means of a bottom-up computation. However, due to the nondeterminism of bottom-up computations of Horn constraint programs, derivable models are not in general unique nor minimal.

Examples. For example, let $P$ be the following Horn constraint program:

$$
P=\{1\{a, b\} \leftarrow\}
$$

Then $\{a\},\{b\}$ and $\{a, b\}$ are its derivable models. The derivable models $\{a\}$ and $\{b\}$ are minimal models of $P$. The third derivable model, $\{a, b\}$, is not a minimal model of $P$.

Since inconsistent monotone constraints may appear in the heads of Horn rules, there are Horn programs $P$ and sets $X \subseteq A t$, such that $T_{P}^{n d}(X)=\emptyset$. Thus, some Horn constraint programs have no computations and no derivable models. However, if a Horn constraint program has models, the existence of computations and derivable models is guaranteed.

To see this, let $M$ be a model of a Horn constraint program $P$. We define a canonical computation $t^{P, M}=\left\langle X_{\alpha}^{P, M}\right\rangle$ by specifying the choice of the next set in the computation in part (2) of Definition 1. Namely, for every ordinal $\beta$, we set

$$
X_{\beta+1}^{P, M}=h \operatorname{set}\left(P\left(X_{\beta}^{P, M}\right)\right) \cap M .
$$

That is, we include in $X_{\alpha}^{P, M}$ all those atoms occurring in the heads of $X_{\beta}^{P, M}$-applicable rules that belong to $M$. We denote the result of $t^{P, M}$ by $\operatorname{Can}(P, M)$. Canonical computations are indeed $P$-computations.

Proposition 2. Let $P$ be a Horn constraint program. If $M \subseteq$ At is a model of $P$, the sequence $t^{P, M}$ is a P-computation.

Proof. As $P$ and $M$ are fixed, to simplify the notation in the proof we will write $X_{\alpha}$ instead of $X_{\alpha}^{P, M}$.

To prove the assertion, it suffices to show that (1) $h \operatorname{set}\left(P\left(X_{\alpha}\right)\right) \cap M \in T_{P}^{n d}\left(X_{\alpha}\right)$, and (2) $X_{\alpha} \subseteq h \operatorname{set}\left(P\left(X_{\alpha}\right)\right) \cap M$, for every ordinal $\alpha$.

(1) Let $X \subseteq M$ and $r \in P(X)$. Since all constraints in $b d(r)$ are monotone, and $X \models b d(r), M \models$ $b d(r)$, as well. From the fact that $M$ is a model of $P$ it follows now that $M \models h d(r)$. Consequently, $M \cap h \operatorname{set}(P(X)) \models h d(r)$ for every $r \in P(X)$. Since $M \cap h \operatorname{set}(P(X)) \subseteq h \operatorname{set}(P(X))$,

$$
M \cap h \operatorname{set}(P(X)) \in T_{P}^{n d}(X) .
$$


Directly from the definition of the canonical computation for $P$ and $M$ we obtain that for every ordinal $\alpha, X_{\alpha} \subseteq M$. Thus, (1), follows.

(2) We proceed by induction. The basis is evident as $X_{0}=\emptyset$. Let us consider an ordinal $\alpha>0$ and let us assume that (2) holds for every ordinal $\beta<\alpha$. If $\alpha=\beta+1$, then $X_{\alpha}=X_{\beta+1}=$ $h \operatorname{set}\left(P\left(X_{\beta}\right)\right) \cap M$. Thus, by the induction hypothesis, $X_{\beta} \subseteq X_{\alpha}$. Since $P$ is a Horn constraint program, it follows that $P\left(X_{\beta}\right) \subseteq P\left(X_{\alpha}\right)$. Thus

$$
X_{\alpha}=X_{\beta+1}=h \operatorname{set}\left(P\left(X_{\beta}\right)\right) \cap M \subseteq h \operatorname{set}\left(P\left(X_{\alpha}\right)\right) \cap M .
$$

If $\alpha$ is a limit ordinal then for every $\beta<\alpha, X_{\beta} \subseteq X_{\alpha}$ and, as before, also $P\left(X_{\beta}\right) \subseteq P\left(X_{\alpha}\right)$. Thus, by the induction hypothesis for every $\beta<\alpha$,

$$
X_{\beta} \subseteq h \operatorname{set}\left(P\left(X_{\beta}\right)\right) \cap M \subseteq h \operatorname{set}\left(P\left(X_{\alpha}\right)\right) \cap M,
$$

which implies that

$$
X_{\alpha}=\bigcup_{\beta<\alpha} X_{\beta} \subseteq h \operatorname{set}\left(P\left(X_{\alpha}\right)\right) \cap M
$$

Canonical computations have the following fixpoint property.

Proposition 3. Let $P$ be a Horn constraint program. For every model $M$ of $P$, we have

$$
h \operatorname{set}(P(\operatorname{Can}(P, M))) \cap M=\operatorname{Can}(P, M) .
$$

Proof. Let $\alpha$ be the length of the canonical computation $t^{P, M}$. Then, $X_{\alpha+1}^{P, M}=X_{\alpha}^{P, M}=\operatorname{Can}(P, M)$. Since $X_{\alpha+1}=h \operatorname{set}\left(X_{\alpha}\right) \cap M$, the assertion follows.

We now gather properties of derivable models that extend properties of the least model of normal Horn logic programs.

Proposition 4. Let P be a Horn constraint program. Then:

1. For every model $M$ of $P, C a n(P, M)$ is a greatest derivable model of $P$ contained in $M$

2. A model $M$ of $P$ is a derivable model if and only if $M=C a n(P, M)$

3. If $M$ is a minimal model of $P$ then $M$ is a derivable model of $P$.

Proof. (1) Let $M^{\prime}$ be a derivable model of $P$ such that $M^{\prime} \subseteq M$. Let $T=\left\langle X_{\alpha}\right\rangle$ be a $P$-derivation such that $M^{\prime}=R_{t}$. We will prove that for every ordinal $\alpha, X_{\alpha} \subseteq X_{\alpha}^{P, M}$. We proceed by transfinite induction. Since $X_{0}=X_{0}^{P, M}=\emptyset$, the basis for the induction is evident. Let us consider an ordinal $\alpha>0$ and assume that for every ordinal $\beta<\alpha, X_{\beta} \subseteq X_{\beta}^{P, M}$.

If $\alpha=\beta+1$, then $X_{\alpha} \in T_{P}^{n d}\left(X_{\beta}\right)$ and so, $X_{\alpha} \subseteq h \operatorname{set}\left(P\left(X_{\beta}\right)\right)$. By the induction hypothesis and by the monotonicity of the constraints in the bodies of rules in $P, X_{\alpha} \subseteq \operatorname{hset}\left(P\left(X_{\beta}^{P, M}\right)\right)$. Thus, since $X_{\alpha} \subseteq R_{t}=M^{\prime} \subseteq M$,

$$
X_{\alpha} \subseteq h \operatorname{set}\left(P\left(X_{\beta}^{P, M}\right)\right) \cap M=X_{\beta+1}^{P, M}=X_{\alpha}^{P, M} .
$$


The case when $\alpha$ is a limit ordinal is straightforward as $X_{\alpha}=\bigcup_{\beta<\alpha} X_{\beta}$ and $X_{\alpha}^{P, M}=\bigcup_{\beta<\alpha} X_{\beta}^{P, M}$. (2) $(\Leftarrow)$ If $M=\operatorname{Can}(P, M)$, then $M$ is the result of the canonical $P$-derivation for $P$ and $M$. In particular, $M$ is a derivable model of $P$.

$(\Rightarrow)$ if $M$ is a derivable model of $P$, then $M$ is also a model of $P$. From (1) it follows that $\operatorname{Can}(P, M)$ is the greatest derivable model of $P$ contained in $M$. Since $M$ itself is derivable, $M=\operatorname{Can}(P, M)$.

(3) From (1) it follows that $\operatorname{Can}(P, M)$ is a derivable model of $P$ and that $\operatorname{Can}(P, M) \subseteq M$. Since $M$ is a minimal model, $\operatorname{Can}(P, M)=M$ and, by (2), $M$ is a derivable model of $P$.

\subsection{Stable Models}

In this section, we will recall and adapt to our setting the definition of stable models proposed and studied by Marek and Truszczyński (2004) and Marek et al. $(2004,2006)$ Let $P$ be a monotoneconstraint program and $M$ a subset of $A t(P)$. The reduct of $P$, denoted by $P^{M}$, is a program obtained from $P$ by:

1. removing from $P$ all rules whose body contains a literal $\operatorname{not}(B)$ such that $M \models B$;

2. removing literals $\operatorname{not}(B)$ for the bodies of the remaining rules.

The reduct of a monotone-constraint program is Horn since it contains no occurrences of default negation. Therefore, the following definition is sound.

Definition 2. Let $P$ be a monotone-constraint program. A set of atoms $M$ is a stable model of $P$ if $M$ is a derivable model of $P^{M}$. We denote the set of stable models of $P$ by $S t(P)$.

The definitions of the reduct and stable models follow and generalize those proposed for normal logic programs, since in the setting of Horn constraint programs, derivable models play the role of a least model.

As in normal logic programming and its standard extensions, stable models of monotoneconstraint programs are supported models and, consequently, models.

Proposition 5. Let $P$ be a monotone-constraint program. If $M \subseteq A t(P)$ is a stable model of $P$, then $M$ is a supported model of $P$.

Proof. Let $M$ be a stable model of $P$. Then, $M$ is a derivable model of $P^{M}$ and, by Proposition 1, $M$ is a supported model of $P^{M}$. It follows that $M$ is a model of $P^{M}$. Directly from the definition of the reduct it follows that $M$ is a model of $P$.

It also follows that $M \subseteq h \operatorname{set}\left(P^{M}(M)\right)$. For every rule $r$ in $P^{M}(M)$, there is a rule $r^{\prime}$ in $P(M)$, which has the same head and the same non-negated literals in the body as $r$. Thus, $h \operatorname{set}\left(P^{M}(M)\right) \subseteq$ $h \operatorname{set}(P(M))$ and, consequently, $M \subseteq h \operatorname{set}(P(M))$. It follows that $M$ is a supported model of $P$.

Examples. Here is an example of stable models of a monotone-constraint program. Let $P$ be a monotone-constraint program that contains the following rules:

$$
\begin{aligned}
& 2\{a, b, c\} \leftarrow 1\{a, d\}, \operatorname{not}(1\{c\}) \\
& 1\{b, c, d\} \leftarrow 1\{a\}, \operatorname{not}(3\{a, b, d\})) \\
& 1\{a\} \leftarrow
\end{aligned}
$$


Let $M=\{a, b\}$. Therefore, $M \not \models 1\{c\}$ and $M \not \forall 3\{a, b, d\}$. Hence the reduct $P^{M}$ contains the following three Horn rules:

$$
\begin{aligned}
& 2\{a, b, c\} \leftarrow 1\{a, d\} \\
& 1\{b, c, d\} \leftarrow 1\{a\} \\
& 1\{a\} \leftarrow
\end{aligned}
$$

Since $M=\{a, b\}$ is a derivable model of $P^{M}, M$ is a stable model of $P$.

Let $M^{\prime}=\{a, b, c\}$. Then $M^{\prime} \models 1\{c\}$ and $M \not \models 3\{a, b, d\}$. Therefore, the reduct $P^{M^{\prime}}$ contains two Horn rules:

$$
\begin{aligned}
& 1\{b, c, d\} \leftarrow 1\{a\} \\
& 1\{a\} \leftarrow
\end{aligned}
$$

Since $M^{\prime}=\{a, b, c\}$ is a derivable models of $P^{M^{\prime}}, M^{\prime}$ is also a stable model of $P$. We note that stable models of a monotone-constraint program, in general, do not form an anti-chain.

If a normal logic program is Horn then its least model is its (only) stable model. Here we have an analogous situation.

Proposition 6. Let $P$ be a Horn monotone-constraint program. Then $M \subseteq A t(P)$ is a derivable model of $P$ if and only if $M$ is a stable model of $P$.

Proof. For every set $M$ of atoms $P=P^{M}$. Thus, $M$ is a derivable model of $P$ if and only if it is a derivable model of $P^{M}$ or, equivalently, a stable model of $P$.

In the next four sections of the paper we show that several fundamental results concerning normal logic programs extend to the class of monotone-constraint programs.

\section{Strong and Uniform Equivalence of Monotone-constraint Programs}

Strong equivalence and uniform equivalence concern the problem of replacing some rules in a logic program with others without changing the overall semantics of the program. More specifically, the strong equivalence concerns replacement of rules within arbitrary programs, and the uniform equivalence concerns replacements of all non-fact rules. In each case, the stipulation is that the resulting program must have the same stable models as the original one. Strong (and uniform) equivalence is an important concept due to its potential uses in program rewriting and optimization.

Strong and uniform equivalence have been studied in the literature mostly for normal logic programs (Lifschitz et al., 2001; Lin, 2002; Turner, 2003; Eiter \& Fink, 2003).

Turner (2003) presented an elegant characterization of strong equivalence of smodels programs, and Eiter and Fink (2003) described a similar characterization of uniform equivalence of normal and disjunctive logic programs. We show that both characterizations can be adapted to the case of monotone-constraint programs. In fact, one can show that under the representations of normal logic programs as monotone-constraint programs (Marek et al., 2004, 2006) our definitions and characterizations of strong and uniform equivalence reduce to those introduced and developed originally for normal logic programs. 


\subsection{M-maximal Models}

A key role in our approach is played by models of Horn constraint programs satisfying a certain maximality condition.

Definition 3. Let $P$ be a Horn constraint program and let $M$ be a model of $P$. A set $N \subseteq M$ such that $N$ is a model of $P$ and $M \cap$ hset $(P(N)) \subseteq N$ is an $M$-maximal model of $P$, written $N \models_{M} P$.

Intuitively, $N$ is an $M$-maximal model of $P$ if $N$ satisfies each rule $r \in P(N)$ "maximally" with respect to $M$. That is, for every $r \in P(N), N$ contains all atoms in $M$ that belong to $h \operatorname{set}(r)$ - the domain of the head of $r$.

To illustrate this notion, let us consider a Horn constraint program $P$ consisting of a single rule:

$$
1\{p, q, r\} \leftarrow 1\{s, t\} .
$$

Let $M=\{p, q, s, t\}$ and $N=\{p, q, s\}$. One can verify that both $M$ and $N$ are models of $P$. Moreover, since the only rule in $P$ is $N$-applicable, and $M \cap\{p, q, r\} \subseteq N, N$ is an $M$-maximal model of $P$. On the other hand, $N^{\prime}=\{p, s\}$ is not $M$-maximal even though $N^{\prime}$ is a model of $P$ and it is contained in $M$.

There are several similarities between properties of models of normal Horn programs and $M$ maximal models of Horn constraint programs. We state and prove here one of them that turns out to be especially relevant to our study of strong and uniform equivalence.

Proposition 7. Let $P$ be a Horn constraint program and let $M$ be a model of $P$. Then $M$ is an $M$-maximal model of $P$ and $C a n(P, M)$ is the least $M$-maximal model of $P$.

Proof. The first claim follows directly from the definition. To prove the second one, we simplify the notation: we will write $N$ for $\operatorname{Can}(P, M)$ and $X_{\alpha}$ for $X_{\alpha}^{P, M}$.

We first show that $N$ is an $M$-maximal model of $P$. Clearly, $N \subseteq M$. Moreover, by Proposition 3, $h \operatorname{set}(P(N)) \cap M=N$. Thus, $N$ is indeed an $M$-maximal model of $P$.

We now show $N$ is the least $M$-maximal model of $P$.

Let $N^{\prime}$ be any $M$-maximal model of $P$. We will show by transfinite induction that $N \subseteq N^{\prime}$. Since $X_{0}=\emptyset$, the basis for the induction holds. Let us consider an ordinal $\alpha>0$ and let us assume that $X_{\beta} \subseteq N^{\prime}$, for every $\beta<\alpha$. To show $N \subseteq N^{\prime}$, it is sufficient to show that $X_{\alpha} \subseteq N^{\prime}$.

Let us assume that $\alpha=\beta+1$ for some $\beta<\alpha$. Then, since $X_{\beta} \subseteq N^{\prime}$ and $P$ is a Horn constraint program, we have $P\left(X_{\beta}\right) \subseteq P\left(N^{\prime}\right)$. Consequently,

$$
X_{\alpha}=X_{\beta+1}=h \operatorname{set}\left(P\left(X_{\beta}\right)\right) \cap M \subseteq h \operatorname{set}\left(P\left(N^{\prime}\right)\right) \cap M \subseteq N^{\prime},
$$

the last inclusion follows from the fact that $N^{\prime}$ is an $M$-maximal model of $P$.

If $\alpha$ is a limit ordinal, then $X_{\alpha}=\bigcup_{\beta<\alpha} X_{\beta}$ and the inclusion $X_{\alpha} \subseteq N^{\prime}$ follows directly from the induction hypothesis.

\subsection{Strong Equivalence and SE-models}

Monotone-constraint programs $P$ and $Q$ are strongly equivalent, denoted by $P \equiv_{s} Q$, if for every monotone-constraint program $R, P \cup R$ and $Q \cup R$ have the same set of stable models.

To study the strong equivalence of monotone-constraint programs, we generalize the concept of an SE-model due to Turner (2003). 
There are close connections between strong equivalence of normal logic programs and the logic here-and-there. The semantics of the logic here-and-there is given in terms of Kripke models with two words which, when rephrased in terms of pairs of interpretations (pairs of sets of propositional atoms), give rise to SE-models.

Definition 4. Let $P$ be a monotone-constraint program and let $X, Y$ be sets of atoms. We say that $(X, Y)$ is an SE-model of $P$ if the following conditions hold: (1) $X \subseteq Y$; (2) $Y \models P$; and (3) $X \models_{Y} P^{Y}$. We denote by $S E(P)$ the set of all SE-models of $P$.

Examples. To illustrate the notion of an SE-model of a monotone-constraint program, let $P$ consist of the following two rules:

$$
\begin{aligned}
& 2\{p, q, r\} \leftarrow 1\{q, r\}, \operatorname{not}(3\{p, q, r\})\} \\
& 1\{p, s\} \leftarrow 1\{p, r\}, \operatorname{not}(2\{p, r\})
\end{aligned}
$$

We observe that $M=\{p, q\}$ is a model of $P$. Let $N=\emptyset$. Then $N \subseteq M$ and $P^{M}(N)$ is empty. It follows that $M \cap h \operatorname{set}\left(P^{M}(N)\right)=\emptyset \subseteq N$ and so, $N \models_{M} P^{M}$. Hence, $(N, M)$ is an SE-models of $P$.

Next, let $N^{\prime}=\{p\}$. It is clear that $N^{\prime} \subseteq M$. Moreover, $P^{M}\left(N^{\prime}\right)=\{1\{p, s\} \leftarrow 1\{p, r\}\}$. Hence $M \cap h \operatorname{set}\left(P^{M}\left(N^{\prime}\right)\right)=\{p\} \subseteq N^{\prime}$ and so, $N^{\prime} \models_{M} P^{M}$. That is, $\left(N^{\prime}, M\right)$ is another SEmodel of $P$.

SE-models yield a simple characterization of strong equivalence of monotone-constraint programs. To state and prove it, we need several auxiliary results.

Lemma 1. Let $P$ be a monotone-constraint program and let $M$ be a model of $P$. Then $(M, M)$ and $\left(C a n\left(P^{M}, M\right), M\right)$ are both SE-models of $P$.

Proof. The requirements (1) and (2) of an SE-model hold for $(M, M)$. Furthermore, since $M$ is a model of $P, M \models P^{M}$. Finally, we also have $h \operatorname{set}(P(M)) \cap M \subseteq M$. Thus, $M \models_{M} P^{M}$.

Similarly, the definition of a canonical computation and Proposition 1, imply the first two requirements of the definition of SE-models for $\left(\operatorname{Can}\left(P^{M}, M\right), M\right)$. The third requirement follows from Proposition 7.

Lemma 2. Let $P$ and $Q$ be two monotone-constraint programs such that $S E(P)=S E(Q)$. Then $\operatorname{St}(P)=\operatorname{St}(Q)$.

Proof. If $M \in S t(P)$, then $M$ is a model of $P$ and, by Lemma $1,(M, M) \in S E(P)$. Hence, $(M, M) \in S E(Q)$ and, in particular, $M \models Q$. By Lemma 1 again,

$$
\left(\operatorname{Can}\left(Q^{M}, M\right), M\right) \in S E(Q) .
$$

By the assumption,

$$
\left(\operatorname{Can}\left(Q^{M}, M\right), M\right) \in S E(P)
$$

and so, $\operatorname{Can}\left(Q^{M}, M\right) \models_{M} P^{M}$ or, in other terms, $\operatorname{Can}\left(Q^{M}, M\right)$ is an $M$-maximal model of $P^{M}$. Since $M \in S t(P), M=\operatorname{Can}\left(P^{M}, M\right)$. By Proposition 7, $M$ is the least $M$-maximal model of $P^{M}$. Thus, $M \subseteq \operatorname{Can}\left(Q^{M}, M\right)$. On the other hand, we have $\operatorname{Can}\left(Q^{M}, M\right) \subseteq M$ and so, $M=\operatorname{Can}\left(Q^{M}, M\right)$. It follows that $M$ is a stable model of $Q$. The other inclusion can be proved in the same way. 
Lemma 3. Let $P$ and $R$ be two monotone-constraint programs. Then $S E(P \cup R)=S E(P) \cap$ $S E(R)$.

Proof. The assertion follows from the following two simple observations. First, for every set $Y$ of atoms, $Y \models(P \cup R)$ if and only if $Y \models P$ and $Y \models R$. Second, for every two sets $X$ and $Y$ of atoms, $X \models_{Y}(P \cup R)^{Y}$ if and only if $X \models_{Y} P^{Y}$ and $X \models_{Y} R^{Y}$.

Lemma 4. Let $P, Q$ be two monotone-constraint programs. If $P \equiv_{s} Q$, then $P$ and $Q$ have the same models.

Proof. Let $M$ be a model of $P$. By $r$ we denote a constraint rule $(M,\{M\}) \leftarrow$. Then, $M \in$ $S t(P \cup\{r\})$. Since $P$ and $Q$ are strongly equivalent, $M \in S t(Q \cup\{r\})$. It follows that $M$ is a model of $Q \cup\{r\}$ and so, also a model of $Q$. The converse inclusion can be proved in the same way.

Theorem 1. Let $P$ and $Q$ be monotone-constraint programs. Then $P \equiv{ }_{s} Q$ if and only if $S E(P)=$ $S E(Q)$.

Proof. $(\Leftarrow)$ Let $R$ be an arbitrary monotone-constraint program. Lemma 3 implies that $S E(P \cup$ $R)=S E(P) \cap S E(R)$ and $S E(Q \cup R)=S E(Q) \cap S E(R)$. Since $S E(P)=S E(Q)$, we have that $S E(P \cup R)=S E(Q \cup R)$. By Lemma 2, $P \cup R$ and $Q \cup R$ have the same stable models. Hence, $P \equiv_{s} Q$ holds.

$(\Rightarrow)$ Let us assume $S E(P) \backslash S E(Q) \neq \emptyset$ and let us consider $(X, Y) \in S E(P) \backslash S E(Q)$. It follows that $X \subseteq Y$ and $Y \models P$. By Lemma $4, Y \models Q$. Since $(X, Y) \notin S E(Q), X \nvdash_{Y} Q^{Y}$. It follows that $X \not \forall Q^{Y}$ or hset $\left(Q^{Y}(X)\right) \cap Y \nsubseteq X$. In the first case, there is a rule $r \in Q^{Y}(X)$ such that $X \not \models h d(r)$. Since $X \subseteq Y$ and $Q^{Y}$ is a Horn constraint program, $r \in Q^{Y}(Y)$. Let us recall that $Y \models Q$ and so, we also have $Y \models Q^{Y}$. It follows that $Y \models h d(r)$. Since $h \operatorname{set}(r) \subseteq h \operatorname{set}\left(Q^{Y}(X)\right)$, $Y \cap h \operatorname{set}\left(Q^{Y}(X)\right) \models h d(r)$. Thus, $h \operatorname{set}\left(Q^{Y}(X)\right) \cap Y \nsubseteq X$ (otherwise, by the monotonicity of $h d(r)$, we would have $X \models h d(r)$ ).

The same property holds in the second case. Thus, it follows that

$$
\left(h \operatorname{set}\left(Q^{Y}(X)\right) \cap Y\right) \backslash X \neq \emptyset .
$$

We define

$$
X^{\prime}=\left(h \operatorname{set}\left(Q^{Y}(X)\right) \cap Y\right) \backslash X .
$$

Let $R$ be a constraint program consisting of the following two rules:

$$
\begin{aligned}
& (X,\{X\}) \leftarrow \\
& (Y,\{Y\}) \leftarrow\left(X^{\prime},\left\{X^{\prime}\right\}\right) .
\end{aligned}
$$

Let us consider a program $Q_{0}=Q \cup R$. Since $Y \models Q$ and $X \subseteq Y, Y \models Q_{0}$. Thus, $Y \models Q_{0}^{Y}$ and, in particular, $\operatorname{Can}\left(Q_{0}^{Y}, Y\right)$ is well defined. Since $R \subseteq Q_{0}^{Y}, X \subseteq \operatorname{Can}\left(Q_{0}^{Y}, Y\right)$. Thus, we have

$$
h \operatorname{set}\left(Q_{0}^{Y}(X)\right) \cap Y \subseteq \operatorname{hset}\left(Q_{0}^{Y}\left(\operatorname{Can}\left(Q_{0}^{Y}, Y\right)\right)\right) \cap Y=\operatorname{Can}\left(Q_{0}^{Y}, Y\right)
$$

(the last equality follows from Proposition 3). We also have $Q \subseteq Q_{0}$ and so,

$$
X^{\prime} \subseteq h \operatorname{set}\left(Q^{Y}(X)\right) \cap Y \subseteq h \operatorname{set}\left(Q_{0}^{Y}(X)\right) \cap Y .
$$


Thus, $X^{\prime} \subseteq \operatorname{Can}\left(Q_{0}^{Y}, Y\right)$. Consequently, by Proposition 3, $Y \subseteq \operatorname{Can}\left(Q_{0}^{Y}, Y\right)$. Since $\operatorname{Can}\left(Q_{0}^{Y}, Y\right)$ $\subseteq Y, Y=\operatorname{Can}\left(Q_{0}^{Y}, Y\right)$ and so, $Y \in S t\left(Q_{0}\right)$.

Since $P$ and $Q$ are strongly equivalent, $Y \in S t\left(P_{0}\right)$, where $P_{0}=P \cup R$. Let us recall that $(X, Y) \in S E(P)$. By Proposition 7, $\operatorname{Can}\left(P^{Y}, Y\right)$ is a least $Y$-maximal model of $P^{Y}$. Since $X$ is a $Y$-maximal model of $P$ (as $X \models_{Y} P^{Y}$ ), it follows that $\operatorname{Can}\left(P^{Y}, Y\right) \subseteq X$. Since $X^{\prime} \nsubseteq X$, $\operatorname{Can}\left(P_{0}^{Y}, Y\right) \subseteq X$. Finally, since $X^{\prime} \subseteq Y, Y \nsubseteq X$. Thus, $Y \neq \operatorname{Can}\left(P_{0}^{Y}, Y\right)$, a contradiction.

It follows that $S E(P) \backslash S E(Q)=\emptyset$. By symmetry, $S E(Q) \backslash S E(P)=\emptyset$, too. Thus, $S E(P)=$ $S E(Q)$.

\subsection{Uniform Equivalence and UE-models}

Let $D$ be a set of atoms. By $r_{D}$ we denote a monotone-constraint rule

$$
r_{D}=(D,\{D\}) \leftarrow .
$$

Adding a rule $r_{D}$ to a program forces all atoms in $D$ to be true (independently of the program).

Monotone-constraint programs $P$ and $Q$ are uniformly equivalent, denoted by $P \equiv_{u} Q$, if for every set of atoms $D, P \cup\left\{r_{D}\right\}$ and $Q \cup\left\{r_{D}\right\}$ have the same stable models.

An SE-model $(X, Y)$ of a monotone-constraint program $P$ is a $U E$-model of $P$ if for every SE-model $\left(X^{\prime}, Y\right)$ of $P$ with $X \subseteq X^{\prime}$, either $X=X^{\prime}$ or $X^{\prime}=Y$ holds. We write $U E(P)$ to denote the set of all UE-models of $P$. Our notion of a UE-model is a generalization of the notion of a UE-model due to Eiter and Fink (2003) to the setting of monotone-constraint programs.

Examples. Let us look again at the program we used to illustrate the concept of an SE-model. We showed there that $(\emptyset,\{p, q\})$ and $(\{p\},\{p, q\})$ are SE-models of $P$. Directly from the definition of UE-models it follows that $(\{p\},\{p, q\})$ is a UE-model of $P$.

We will now present a characterization of uniform equivalence of monotone-constraint programs under the assumption that their sets of atoms are finite. One can prove a characterization of uniform equivalence of arbitrary monotone-constraint programs, generalizing one of the results by Eiter and Fink (2003). However, both the characterization and its proof are more complex and, for brevity, we restrict our attention to the finite case only.

We start with an auxiliary result, which allows us to focus only on atoms in $A t(P)$ when deciding whether a pair $(X, Y)$ of sets of atoms is an SE-model of a monotone-constraint program $P$.

Lemma 5. Let $P$ be a monotone-constraint program, $X \subseteq Y$ two sets of atoms. Then $(X, Y) \in$ $S E(P)$ if and only if $(X \cap A t(P), Y \cap A t(P)) \in S E(P)$.

Proof. Since $X \subseteq Y$ is given, and $X \subseteq Y$ implies $X \cap A t(P) \subseteq Y \cap A t(P)$, the first condition of the definition of an SE-model holds on both sides of the equivalence.

Next, we note that for every constraint $C, Y \models C$ if and only if $Y \cap \operatorname{Dom}(C) \models C$. Therefore, $Y \models P$ if and only if $Y \cap A t(P) \models P$. That is, the second condition of the definition of an SE-model holds for $(X, Y)$ if and only if it holds for $(X \cap A t(P), Y \cap A t(P))$.

Finally, we observe that $P^{Y}=P^{Y \cap A t(P)}$ and $P(X)=P(X \cap A t(P))$. Therefore,

$$
Y \cap h \operatorname{set}\left(P^{Y}(X)\right)=Y \cap h \operatorname{set}\left(P^{Y \cap A t(P)}(X \cap A t(P))\right) .
$$

Since $h \operatorname{set}\left(P^{Y \cap A t(P)}(X \cap A t(P))\right) \subseteq A t(P)$, it follows that

$$
Y \cap h \operatorname{set}\left(P^{Y}(X)\right) \subseteq X
$$


if and only if

$$
Y \cap A t(P) \cap h \operatorname{set}\left(P^{Y \cap A t(P)}(X \cap A t(P))\right) \subseteq X \cap A t(P) .
$$

Thus, $X \models_{Y} P^{Y}$ if and only if $X \cap A t(P) \models_{Y \cap A t(P)} P^{Y \cap A t(P)}$. That is, the third condition of the definition of an SE-model holds for $(X, Y)$ if and only if it holds for $(X \cap A t(P), Y \cap A t(P))$.

Lemma 6. Let $P$ be a monotone-constraint program such that $A t(P)$ is finite. Then for every $(X, Y) \in S E(P)$ such that $X \neq Y$, the set

$$
\left\{X^{\prime}: X \subseteq X^{\prime} \subseteq Y, X^{\prime} \neq Y,\left(X^{\prime}, Y\right) \in S E(P)\right\}
$$

has a maximal element.

Proof. If $A t(P) \cap X=A t(P) \cap Y$, then for every element $y \in Y \backslash X, Y \backslash\{y\}$ is a maximal element of the set (2). Indeed, since $(X, Y) \in S E(P)$, by Lemma 5, $(X \cap A t(P), Y \cap A t(P)) \in S E(P)$. Since $X \cap A t(P)=Y \cap A t(P)$ and $y \notin A t(P), X \cap A t(P)=(Y \backslash\{y\}) \cap A t(P)$. Therefore, $((Y \backslash\{y\}) \cap A t(P), Y \cap A t(P)) \in S E(P)$. Then from Lemma 5 and the fact $Y \backslash\{y\} \subseteq Y$, we have $(Y \backslash\{y\}, Y) \in S E(P)$. Therefore, $Y \backslash\{y\}$ belongs to the set (2) and so, it is a maximal element of this set.

Thus, let us assume that $A t(P) \cap X \neq A t(P) \cap Y$. Let us define $X^{\prime}=X \cup(Y \backslash A t(P))$. Then $X \subseteq X^{\prime} \subseteq Y$ and $X^{\prime} \neq Y$. Moreover, no element in $X^{\prime} \backslash X$ belongs to $\operatorname{At}(P)$. That is, $X^{\prime} \cap A t(P)=X \cap A t(P)$. Thus, by Lemma 5, $\left(X^{\prime}, Y\right) \in S E(P)$ and so, $X^{\prime}$ belongs to the set (2). Since $Y \backslash X^{\prime} \subseteq A t(P)$, by the finiteness of $A t(P)$ it follows that the set (2) contains a maximal element containing $X^{\prime}$. In particular, it contains a maximal element.

Theorem 2. Let $P$ and $Q$ be two monotone-constraint programs such that $A t(P) \cup A t(Q)$ is finite. Then $P \equiv_{u} Q$ if and only if $U E(P)=U E(Q)$.

Proof. $(\Leftarrow)$ Let $D$ be an arbitrary set of atoms and $Y$ be a stable model of $P \cup\left\{r_{D}\right\}$. Then $Y$ is a model of $P \cup\left\{r_{D}\right\}$. In particular, $Y$ is a model of $P$ and so, $(Y, Y) \in U E(P)$. It follows that $(Y, Y) \in U E(Q)$, too. Thus, $Y$ is a model of $Q$. Since $Y$ is a model of $r_{D}, D \subseteq Y$. Consequently, $Y$ is a model of $Q \cup\left\{r_{D}\right\}$ and thus, also of $\left(Q \cup\left\{r_{D}\right\}\right)^{Y}$.

Let $X=\operatorname{Can}\left(\left(Q \cup\left\{r_{D}\right\}\right)^{Y}, Y\right)$. Then $D \subseteq X \subseteq Y$ and, by Proposition 7, $X$ is a $Y$-maximal model of $\left(Q \cup\left\{r_{D}\right\}\right)^{Y}$. Consequently, $X$ is a $Y$-maximal model of $Q^{Y}$. Since $X \subseteq Y$ and $Y \models Q$, $(X, Y) \in S E(Q)$.

Let us assume that $X \neq Y$. Then, by Lemma 6, there is a maximal set $X^{\prime}$ such that $X \subseteq X^{\prime} \subseteq$ $Y, X^{\prime} \neq Y$ and $\left(X^{\prime}, Y\right) \in S E(Q)$. It follows that $\left(X^{\prime}, Y\right) \in U E(Q)$. Thus, $\left(X^{\prime}, Y\right) \in U E(P)$ and so, $X^{\prime} \models_{Y} P^{Y}$. Since $D \subseteq X^{\prime}, X^{\prime} \models_{Y}\left(P \cup\left\{r_{D}\right\}\right)^{Y}$. We recall that $Y$ is a stable model of $P \cup\left\{r_{D}\right\}$. Thus, $Y=\operatorname{Can}\left(\left(P \cup\left\{r_{D}\right\}\right)^{Y}, Y\right)$. By Proposition $7, Y \subseteq X^{\prime}$ and so we get $X^{\prime}=Y$, a contradiction. It follows that $X=Y$ and, consequently, $Y$ is a stable model of $Q \cup\left\{r_{D}\right\}$.

By symmetry, every stable model of $Q \cup\left\{r_{D}\right\}$ is also a stable model of $P \cup\left\{r_{D}\right\}$. $(\Rightarrow)$ First, we note that $(Y, Y) \in U E(P)$ if and only if $Y$ is a model of $P$. Next, we note that $P$ and $Q$ have the same models. Indeed, the argument used in the proof of Lemma 4 works also under the assumption that $P \equiv_{u} Q$. Thus, $(Y, Y) \in U E(P)$ if and only if $(Y, Y) \in U E(Q)$.

Now let us assume that $U E(P) \neq U E(Q)$. Let $(X, Y)$ be an element of $(U E(P) \backslash U E(Q)) \cup$ $(U E(Q) \backslash U E(P))$. Without loss of generality, we can assume that $(X, Y) \in U E(P) \backslash U E(Q)$. Since $(X, Y) \in U E(P)$, it follows that 
1. $X \subseteq Y$

2. $Y \models P$ and, consequently, $Y \models Q$

3. $X \neq Y$ (otherwise, by our earlier observations, $(X, Y)$ would belong to $U E(Q)$ ).

Let $R=\left(Q \cup\left\{r_{X}\right\}\right)^{Y}$. Clearly, $R$ is a Horn constraint program. Moreover, since $Y \models Q$ and $X \subseteq Y, Y \models R$. Thus, $\operatorname{Can}(R, Y)$ is defined. We have $X \subseteq \operatorname{Can}(R, Y) \subseteq Y$. We claim that $\operatorname{Can}(R, Y) \neq Y$. Let us assume to the contrary that $\operatorname{Can}(R, Y)=Y$. Then $Y \in \operatorname{St}\left(Q \cup\left\{r_{X}\right\}\right)$. Hence, $Y \in S t\left(P \cup\left\{r_{X}\right\}\right)$, that is, $Y=\operatorname{Can}\left(\left(P \cup\left\{r_{X}\right\}\right)^{Y}, Y\right)$. By Proposition 7, $Y$ is the least $Y$-maximal model of $\left(P \cup\left\{r_{X}\right\}\right)^{Y}$ and $X$ is a $Y$-maximal model of $\left(P \cup\left\{r_{X}\right\}\right)^{Y}$ (since $(X, Y) \in S E(P), X \models_{Y} P^{Y}$ and so, $X \models_{Y}\left(P \cup\left\{r_{X}\right\}\right)^{Y}$, too). Consequently, $Y \subseteq X$ and, as $X \subseteq Y, X=Y$, a contradiction.

Thus, $\operatorname{Can}(R, Y) \neq Y$. By Proposition 7, $\operatorname{Can}(R, Y)$ is a $Y$-maximal model of $R$. Since $Q^{Y} \subseteq R$, it follows that $\operatorname{Can}(R, Y)$ is a $Y$-maximal model of $Q^{Y}$ and so, $(\operatorname{Can}(R, Y), Y) \in$ $S E(Q)$. Since $\operatorname{Can}(R, Y) \neq Y$, from Lemma 6 it follows that there is a maximal set $X^{\prime}$ such that $\operatorname{Can}(R, Y) \subseteq X^{\prime} \subseteq Y, X^{\prime} \neq Y$ and $\left(X^{\prime}, Y\right) \in S E(Q)$. By the definition, $\left(X^{\prime}, Y\right) \in U E(Q)$. Since $(X, Y) \notin U E(Q) . X \neq X^{\prime}$. Consequently, since $X \subseteq X^{\prime}, X^{\prime} \neq Y$ and $(X, Y) \in U E(P)$, $\left(X^{\prime}, Y\right) \notin U E(P)$.

Thus, $\left(X^{\prime}, Y\right) \in U E(Q) \backslash U E(P)$. By applying now the same argument as above to $\left(X^{\prime}, Y\right)$ we show the existence of $X^{\prime \prime}$ such that $X^{\prime} \subseteq X^{\prime \prime} \subseteq Y, X^{\prime} \neq X^{\prime \prime}, X^{\prime \prime} \neq Y$ and $\left(X^{\prime \prime}, Y\right) \in S E(P)$. Consequently, we have $X \subseteq X^{\prime \prime}, X \neq X^{\prime \prime}$ and $Y \neq X^{\prime \prime}$, which contradicts the fact that $(X, Y) \in$ $U E(P)$. It follows then that $U E(P)=U E(Q)$.

Examples. Let $P=\{1\{p, q\} \leftarrow \operatorname{not}(2\{p, q\})\}$, and $Q=\{p \leftarrow \operatorname{not}(q), q \leftarrow \operatorname{not}(p)\}$. Then $P$ and $Q$ are strongly equivalent. We note that both programs have $\{p\},\{q\}$, and $\{p, q\}$ as models. Furthermore, $(\{p\},\{p\}),(\{q\},\{q\}),(\{p\},\{p, q\}),(\{q\},\{p, q\}),(\{p, q\},\{p, q\})$ and $(\emptyset,\{p, q\})$ are "all" SE-models of the two programs ${ }^{3}$.

Thus, by Theorem 1, $P$ and $Q$ are strongly equivalent.

We also observe that the first five SE-models are precisely UE-models of $P$ and $Q$. Therefore, by Theorem 2, $P$ and $Q$ are also uniformly equivalent.

It is possible for two monotone-constraint programs to be uniformly but not strongly equivalent. If we add rule $p \leftarrow$ to $P$, and rule $p \leftarrow q$ to $Q$, then the two resulting programs, say $P^{\prime}$ and $Q^{\prime}$, are uniformly equivalent. However, they are not strongly equivalent. The programs $P^{\prime} \cup\{q \leftarrow p\}$ and $Q^{\prime} \cup\{q \leftarrow p\}$ have different stable models. Another way to show it is by observing that $(\emptyset,\{p, q\})$ is an SE-model of $Q^{\prime}$ but not an SE-model of $P^{\prime}$.

\section{Fages Lemma}

In general, supported models and stable models of a logic program (both in the normal case and the monotone-constraint case) do not coincide. Fages Lemma (Fages, 1994), later extended by Erdem and Lifschitz (2003), establishes a sufficient condition under which a supported model of a normal logic program is stable. In this section, we show that Fages Lemma extends to programs with monotone constraints.

3. From Lemma 5 and Theorem 1, it follows that only those SE-models that contain atoms only from $A t(P) \cup A t(Q)$ are the essential ones. 
Definition 5. A monotone-constraint program $P$ is called tight on a set $M \subseteq A t(P)$ of atoms, if there exists a mapping $\lambda$ from $M$ to ordinals such that for every rule $A \leftarrow A_{1}, \ldots, A_{k}, \operatorname{not}\left(A_{k+1}\right)$, $\ldots, \operatorname{not}\left(A_{m}\right)$ in $P(M)$, if $X$ is the domain of $A$ and $X_{i}$ the domain of $A_{i}, 1 \leq i \leq k$, then for every $x \in M \cap X$ and for every $a \in M \cap \bigcup_{i=1}^{k} X_{i}, \lambda(a)<\lambda(x)$.

We will now show that tightness provides a sufficient condition for a supported model to be stable. In order to prove a general result, we first establish it in the Horn case.

Lemma 7. Let $P$ be a Horn monotone-constraint program and let $M$ be a supported model of $P$. If $P$ is tight on $M$, then $M$ is a stable model of $P$.

Proof. Let $M$ be an arbitrary supported model of $P$ such that $P$ is tight on $M$. Let $\lambda$ be a mapping showing the tightness of $P$ on $M$. We will show that for every ordinal $\alpha$ and for every atom $x \in M$ such that $\lambda(x) \leq \alpha, x \in \operatorname{Can}(P, M)$. We will proceed by induction.

For the basis of the induction, let us consider an atom $x \in M$ such that $\lambda(x)=0$. Since $M$ is a supported model for $P$ and $x \in M$, there exists a rule $r \in P(M)$ such that $x \in h$ set $(r)$. Moreover, since $P$ is tight on $M$, for every $A \in b d(r)$ and for every $y \in \operatorname{Dom}(A) \cap M, \lambda(y)<\lambda(x)=0$. Thus, for every $A \in b d(r), \operatorname{Dom}(A) \cap M=\emptyset$. Since $M \models b d(r)$ and since $P$ is a Horn monotoneconstraint program, it follows that $\emptyset \models b d(r)$. Consequently, hset $(r) \cap M \subseteq \operatorname{Can}(P, M)$ and so, $x \in \operatorname{Can}(P, M)$.

Let us assume that the assertion holds for every ordinal $\beta<\alpha$ and let us consider $x \in M$ such that $\lambda(x)=\alpha$. As before, since $M$ is a supported model of $P$, there exists a rule $r \in P(M)$ such that $x \in h$ set $(r)$. By the assumption, $P$ is tight on $M$ and, consequently, for every $A \in b d(r)$ and for every $y \in \operatorname{Dom}(A) \cap M, \lambda(y)<\lambda(x)=\alpha$. By the induction hypothesis, for every $A \in b d(r)$, $\operatorname{Dom}(A) \cap M \subseteq \operatorname{Can}(P, M)$. Since $P$ is a Horn monotone-constraint program, $\operatorname{Can}(P, M) \models$ $b d(r)$. By Proposition 3, hset $(r) \cap M \subseteq \operatorname{Can}(P, M)$ and so, $x \in \operatorname{Can}(P, M)$.

It follows that $M \subseteq \operatorname{Can}(P, M)$. By the definition of a canonical computation, we have $\operatorname{Can}(P, M) \subseteq M$. Thus, $M=\operatorname{Can}(P, M)$. By Proposition 6, $M$ is a stable model of $P$.

Given this lemma, the general result follows easily.

Theorem 3. Let $P$ be a monotone-constraint program and let $M$ be a supported model of $P$. If $P$ is tight on $M$, then $M$ is a stable model of $P$.

Proof. One can check that if $M$ is a supported model of $P$, then it is a supported model of the reduct $P^{M}$. Since $P$ is tight on $M$, the reduct $P^{M}$ is tight on $M$, too. Thus, $M$ is a stable model of $P^{M}$ (by Lemma 7) and, consequently, a derivable model of $P^{M}$ (by Proposition 6). It follows that $M$ is a stable model of $P$.

\section{Logic $P L^{m c}$ and the Completion of a Monotone-constraint Program}

The completion of a normal logic program (Clark, 1978) is a propositional theory whose models are precisely supported models of the program. Thus, supported models of normal logic programs can be computed by means of SAT solvers. Under some conditions, for instance, when the assumptions of Fages Lemma hold, supported models are stable. Thus, computing models of the completion can yield stable models, an idea implemented in the first version of cmodels software (Babovich \& Lifschitz, 2002). 
Our goal is to extend the concept of the completion to programs with monotone constraints. The completion, as we define it, retains much of the structure of monotone-constraint rules and allow us, in the restricted setting of lparse programs, to use pseudo-boolean constraint solvers to compute supported models of such programs. In this section we define the completion and prove a result relating supported models of programs to models of the completion. We discuss extensions of this result in the next section and their practical computational applications in Section 8.

To define the completion, we first introduce an extension of propositional logic with monotone constraints, a formalism we denote by $P L^{m c}$. A formula in the logic $P L^{m c}$ is an expression built from monotone constraints by means of boolean connectives $\wedge, \vee$ (and their infinitary counterparts), $\rightarrow$ and $\neg$. The notion of a model of a constraint, which we discussed earlier, extends in a standard way to the class of formulas in the logic $P L^{m c}$.

For a set $L=\left\{A_{1}, \ldots, A_{k}, \operatorname{not}\left(A_{k+1}\right), \ldots, \operatorname{not}\left(A_{m}\right)\right\}$ of literals, we define

$$
L^{\wedge}=A_{1} \wedge \ldots \wedge A_{k} \wedge \neg A_{k+1} \wedge \ldots \wedge \neg A_{m} .
$$

Let $P$ be a monotone-constraint program. We form the completion of $P$, denoted $\operatorname{Comp}(P)$, as follows:

1. For every rule $r \in P$ we include in $\operatorname{Comp}(P)$ a $P L^{m c}$ formula

$$
[b d(r)]^{\wedge} \rightarrow h d(r)
$$

2. For every atom $x \in A t(P)$, we include in $\operatorname{Comp}(P)$ a $P L^{m c}$ formula

$$
x \rightarrow \bigvee\left\{[b d(r)]^{\wedge}: r \in P, x \in h \operatorname{set}(r)\right\}
$$

(we note that when the set of rules in $P$ is infinite, the disjunction may be infinitary).

The following theorem generalizes a fundamental result on the program completion from normal logic programming (Clark, 1978) to the case of programs with monotone constraints.

Theorem 4. Let $P$ be a monotone-constraint program. A set $M \subseteq A t(P)$ is a supported model of $P$ if and only if $M$ is a model of $\operatorname{Comp}(P)$.

Proof. $(\Rightarrow)$ Let us suppose that $M$ is a supported model of $P$. Then $M$ is a model of $P$, that is, for each rule $r \in P$, if $M \models b d(r)$ then $M \models h d(r)$. Since $M \models b d(r)$ if and only if $M \models[b d(r)]^{\wedge}$, it follows that all formulas in $\operatorname{Comp}(P)$ of the first type are satisfied by $M$.

Moreover, since $M$ is a supported model of $P, M \subseteq h \operatorname{set}(P(M))$. That is, for every atom $x \in M$, there exists at least one rule $r$ in $P$ such that $x \in h \operatorname{set}(r)$ and $M \models b d(r)$. Therefore, all formulas in $\operatorname{Comp}(P)$ of the second type are satisfied by $M$, too.

$(\Leftarrow)$ Let us now suppose that $M$ is a model of $\operatorname{Comp}(P)$. Since $M \models b d(r)$ if and only if $M \models$ $[b d(r)]^{\wedge}$, and since $M$ satisfies formulas of the first type in $\operatorname{Comp}(P), M$ is a model of $P$.

Let $x \in M$. Since $M$ satisfies the formula $x \rightarrow \bigvee\left\{[b d(r)]^{\wedge}: r \in P, x \in h s e t(r)\right\}$, it follows that $M$ satisfies $\bigvee\left\{[b d(r)]^{\wedge}: r \in P, x \in h \operatorname{set}(r)\right\}$. That is, there is $r \in P$ such that $M$ satisfies $[b d(r)]^{\wedge}$ (and so, $b d(r)$, too) and $x \in h \operatorname{set}(r)$. Thus, $x \in h \operatorname{set}(P(M))$. Hence, $M$ is a supported model of $P$.

Theorems 3 and 4 have the following corollary. 
Corollary 5. Let $P$ be a monotone-constraint program. A set $M \subseteq A t(P)$ is a stable model of $P$ if $P$ is tight on $M$ and $M$ is a model of $\operatorname{Comp}(P)$.

We observe that for the material in this section it is not necessary to require that constraints appearing in the bodies of program rules be monotone. However, since we are only interested in this case, we adopted the monotonicity assumption here, as well.

\section{Loops and Loop Formulas in Monotone-constraint Programs}

The completion alone is not quite satisfactory as it relates supported not stable models of monotoneconstraint programs with models of $P L^{m c}$ theories. Loop formulas, proposed by Lin and Zhao (2002), provide a way to eliminate those supported models of normal logic programs, which are not stable. Thus, they allow us to use SAT solvers to compute stable models of arbitrary normal logic programs and not only those, for which supported and stable models coincide.

We will now extend this idea to monotone-constraint programs. In this section, we will restrict our considerations to programs $P$ that are finitary, that is, $A t(P)$ is finite. This restriction implies that monotone constraints that appear in finitary programs have finite domains.

Let $P$ be a finitary monotone-constraint program. The positive dependency graph of $P$ is the directed graph $G_{P}=(V, E)$, where $V=A t(P)$ and $\langle u, v\rangle$ is an edge in $E$ if there exists a rule $r \in P$ such that $u \in h \operatorname{set}(r)$ and $v \in \operatorname{Dom}(A)$ for some monotone constraint $A \in b d(r)$ (that is, $A$ appears non-negated in $b d(r))$. We note that positive dependency graphs of finitary programs are finite.

Let $G=(V, E)$ be a directed graph. A set $L \subseteq V$ is a loop in $G$ if the subgraph of $G$ induced by $L$ is strongly connected. A loop is maximal if it is not a proper subset of any other loop in $G$. Thus, maximal loops are vertex sets of strongly connected components of $G$. A maximal loop is terminating if there is no edge in $G$ from $L$ to any other maximal loop.

These concepts can be extended to the case of programs. By a loop (maximal loop, terminating loop) of a monotone-constraint program $P$, we mean the loop (maximal loop, terminating loop) of the positive dependency graph $G_{P}$ of $P$. We observe that every finitary monotone-constraint program $P$ has a terminating loop, since $G_{P}$ is finite.

Let $X \subseteq A t(P)$. By $G_{P}[X]$ we denote the subgraph of $G_{P}$ induced by $X$. We observe that if $X \neq \emptyset$ then every loop of $G_{P}[X]$ is a loop of $G_{P}$.

Let $P$ be a monotone-constraint program $P$. For every model $M$ of $P$ (in particular, for every model $M$ of $\operatorname{Comp}(P)$ ), we define $M^{-}=M \backslash \operatorname{Can}\left(P^{M}, M\right)$. Since $M$ is a model of $P, M$ is a model of $P^{M}$. Thus, $\operatorname{Can}\left(P^{M}, M\right)$ is well defined and so is $M^{-}$.

For every loop in the graph $G_{P}$ we will now define the corresponding loop formula. First, for a constraint $A=(X, C)$ and a set $L \subseteq A t$, we set $A_{\mid L}=(X,\{Y \in C: Y \cap L=\emptyset\})$ and call $A_{\mid L}$ the restriction of $A$ to $L$. Next, let $r$ be a monotone-constraint rule, say

$$
r=A \leftarrow A_{1}, \ldots, A_{k}, \operatorname{not}\left(A_{k+1}\right), \ldots, \operatorname{not}\left(A_{m}\right) .
$$

If $L \subseteq A t$, then define a $P L^{m c}$ formula $\beta_{L}(r)$ by setting

$$
\beta_{L}(r)=A_{1 \mid L} \wedge \ldots \wedge A_{k \mid L} \wedge \neg A_{k+1} \wedge \ldots \wedge \neg A_{m} .
$$

Let $L$ be a loop of a monotone-constraint program $P$. Then, the loop formula for $L$, denoted by $L P(L)$, is the $P L^{m c}$ formula

$$
L P(L)=\bigvee L \rightarrow \bigvee\left\{\beta_{L}(r): r \in P \text { and } L \cap h \operatorname{set}(r) \neq \emptyset\right\}
$$


(we recall that we use the convention to write $a$ for the constraint $C(a)=(\{a\},\{\{a\}\})$. A loop completion of a finitary monotone-constraint program $P$ is the $P L^{m c}$ theory

$$
\operatorname{LComp}(P)=\operatorname{Comp}(P) \cup\left\{L P(L): L \text { is a loop in } G_{P}\right\}
$$

The following theorem exploits the concept of a loop formula to provide a necessary and sufficient condition for a model being a stable model. transfinite one.

Theorem 6. Let $P$ be a finitary monotone-constraint program. A set $M \subseteq A t(P)$ is a stable model of $P$ if and only if $M$ is a model of $\operatorname{LComp}(P)$.

Proof. $(\Rightarrow)$ Let $M$ be a stable model of $P$. Then $M$ is a supported model of $P$ and, by Theorem 4, $M \models \operatorname{Comp}(P)$.

Let $L$ be a loop in $P$. If $M \cap L=\emptyset$ then $M \models L P(L)$. Thus, let us assume that $M \cap L \neq \emptyset$. Since $M$ is a stable model of $P, M$ is a derivable model of $P^{M}$, that is, $M=\operatorname{Can}\left(P^{M}, M\right)$. Let $\left(X_{n}\right)_{n=0,1, \ldots}$ be the canonical $P^{M}$-derivation with respect to $M$ (since we assume that $P$ is finite and each constraint in $P$ has a finite domain, $P$-derivations reach their results in finitely many steps). Since $\operatorname{Can}\left(P^{M}, M\right) \cap L=M \cap L \neq \emptyset$, there is a smallest index $n$ such that $X_{n} \cap L \neq \emptyset$. In particular, it follows that $n>0$ (as $X_{0}=\emptyset$ ) and $L \cap X_{n-1}=\emptyset$.

Since $X_{n}=h \operatorname{set}\left(P^{M}\left(X_{n-1}\right) \cap M\right.$ and $X_{n} \cap L \neq \emptyset$, there is a rule $r \in P^{M}\left(X_{n-1}\right)$ such that $h \operatorname{set}(r) \cap L \neq \emptyset$, that is, such that $L \cap h \operatorname{set}(r)) \neq \emptyset$. Let $r^{\prime}$ be a rule in $P$, which contributes $r$ to $P^{M}$. Then, for every literal $\operatorname{not}(A) \in b d\left(r^{\prime}\right), M \models \operatorname{not}(A)$. Let $A \in b d\left(r^{\prime}\right)$. Then $A \in b d(r)$ and so, $X_{n-1} \models A$. Since $X_{n-1} \cap L=\emptyset, X_{n-1} \models A_{\mid L}$, too, By the monotonicity of $A_{\mid L}, M \models A_{\mid L}$. Thus, $M \models \beta_{L}\left(r^{\prime}\right)$. Since $\left.h \operatorname{set}\left(r^{\prime}\right) \cap L \neq \emptyset, L \cap h \operatorname{set}(r)\right) \neq \emptyset$ and so, $M \models L P(L)$. Thus, $M \models L \operatorname{Comp}(P)$.

$(\Leftarrow)$ Let us consider a set $M \subseteq A t(P)$ such that $M$ is not a stable model of $P$. If $M$ is not a supported model of $P$ that $M \not \models \operatorname{Comp}(P)$ and so $M$ is not a model of $L C o m p(P)$. Thus, let us assume that $M$ is a supported model of $P$. It follows that $M^{-} \neq \emptyset$. Let $L \subseteq M^{-}$be a terminating loop for $G_{P}\left[M^{-}\right]$.

Let $r^{\prime}$ be an arbitrary rule in $P$ such that $\left.L \cap h \operatorname{set}\left(r^{\prime}\right)\right) \neq \emptyset$, and let $r$ be the rule obtained from $r^{\prime}$ by removing negated constraints from its body. Now, let us assume that $M \models \beta_{r^{\prime}}(L)$. It follows that for every literal $\operatorname{not}(A) \in b d\left(r^{\prime}\right), M \models \operatorname{not}(A)$. Thus, $r \in P^{M}$. Moreover, since $L$ is a terminating loop for $G_{P}\left[M^{-}\right]$, for every constraint $A \in b d\left(r^{\prime}\right), \operatorname{Dom}(A) \cap M^{-} \subseteq L$. Since $M \models A_{\mid L}$, it follows that $\operatorname{Can}\left(P^{M}, M\right) \models A$. Consequently, hset $\left(r^{\prime}\right) \cap L \subseteq h \operatorname{set}\left(r^{\prime}\right) \cap M \subseteq \operatorname{Can}\left(P^{M}, M\right)$ and so, $L \cap \operatorname{Can}\left(P^{M}, M\right) \neq \emptyset$, a contradiction. Thus, $M \forall \vee \bigvee\left\{\beta_{r^{\prime}}(L): r^{\prime} \in P\right.$ and $\left.\left.L \cap h \operatorname{set}\left(r^{\prime}\right)\right) \neq \emptyset\right\}$. Since $M \models \bigvee L$, it follows that $M \not \models L P(L)$ and so, $M \not \models L C o m p(P)$.

The following result follows directly from the proof of Theorem 6 and provides us with a way to filter out specific non-stable supported models from $\operatorname{Comp}(P)$.

Theorem 7. Let $P$ be a finitary monotone-constraint program and $M$ a model of $C o m p(P)$. If $M^{-}$ is not empty, then $M$ violates the loop formula of every terminating loop of $G_{P}\left[M^{-}\right]$.

Finally, we point out that, Theorem 6 does not hold when a program $P$ contains infinitely many rules. Here is a counterexample:

Examples. Let $P$ be the set of following rules: 


$$
\begin{aligned}
& 1\left\{a_{0}\right\} \leftarrow 1\left\{a_{1}\right\} \\
& 1\left\{a_{1}\right\} \leftarrow 1\left\{a_{2}\right\} \\
& \cdots \\
& 1\left\{a_{n}\right\} \leftarrow 1\left\{a_{n+1}\right\} \\
& \ldots
\end{aligned}
$$

Let $M=\left\{a_{0}, \ldots, a_{n}, \ldots\right\}$. Then $M$ is a supported model of $P$. The only stable model of $P$ is $\emptyset$. However, $M^{-}=M \backslash \emptyset$ does not contain any terminating loop. The problem arises because there is an infinite simple path in $G_{P}\left[M^{-}\right]$. Therefore, $G_{P}\left[M^{-}\right]$does not have a sink, yet it does not have a terminating loop either.

The results of this section, concerning the program completion and loop formulas - most importantly, the loop-completion theorem - form the basis of a new software system to compute stable models of lparse programs. We discuss this matter in Section 8.

\section{Programs with Convex Constraints}

We will now discuss programs with convex constraints, which are closely related to programs with monotone constraints. Programs with convex constraints are of interest as they do not involve explicit occurrences of the default negation operator not, yet are as expressive as programs with monotone-constraints. Moreover, they directly subsume an essential fragment of the class of lparse programs (Simons et al., 2002).

A constraint $(X, C)$ is convex, if for every $W, Y, Z \subseteq X$ such that $W \subseteq Y \subseteq Z$ and $W, Z \in C$, we have $Y \in C$. A constraint rule of the form (1) is a convex-constraint rule if $A, A_{1}, \ldots, A_{n}$ are convex constraints and $m=k$. Similarly, a constraint program built of convex-constraint rules is a convex-constraint program.

The concept of a model discussed in Section 2 applies to convex-constraint programs. To define supported and stable models of convex-constraint programs, we view them as special programs with monotone-constraints.

To this end, we define the upward and downward closures of a constraint $A=(X, C)$ to be constraints $A^{+}=\left(X, C^{+}\right)$and $A^{-}=\left(X, C^{-}\right)$, respectively, where

$$
\begin{aligned}
& C^{+}=\{Y \subseteq X: \text { for some } W \in C, W \subseteq Y\}, \text { and } \\
& C^{-}=\{Y \subseteq X: \text { for some } W \in C, Y \subseteq W\} .
\end{aligned}
$$

We note that the constraint $A^{+}$is monotone. We call a constraint $(X, C)$ antimonotone if $C$ is closed under subset, that is, for every $W, Y \subseteq X$, if $Y \in C$ and $W \subseteq Y$ then $W \in C$. It is clear that the constraint $A^{-}$is antimonotone.

The upward and downward closures allow us to represent any convex constraint as the "conjunction" of a monotone constraint and an antimonotone constraint.Namely, we have the following property of convex constraints.

Proposition 8. A constraint $(X, C)$ is convex if and only if $C=C^{+} \cap C^{-}$.

Proof. $(\Leftarrow)$ Let us assume that $C=C^{+} \cap C^{-}$and let us consider a set $M$ such that $M^{\prime} \subseteq M \subseteq M^{\prime \prime}$, where $M^{\prime}, M^{\prime \prime} \in C$. it follows that $M^{\prime} \in C^{+}$and $M^{\prime \prime} \in C^{-}$. Thus, $M \in C^{+}$and $M \in C^{-}$. Consequently, $M \in C$, which implies that $(X, C)$ is convex. 
$(\Rightarrow)$ The definitions directly imply that $C \subseteq C^{+}$and $C \subseteq C^{-}$. Thus, $C \subseteq C^{+} \cap C^{-}$. Let us consider $M \in C^{+} \cap C^{-}$. Then there are sets $M^{\prime}, M^{\prime \prime} \in C$ such that $M^{\prime} \subseteq M$ and $M \subseteq M^{\prime \prime}$. Since $C$ is convex, $M \in C$. Thus, $C^{+} \cap C^{-} \subseteq C$ and so, $C=C^{+} \cap C^{-}$.

Proposition 8 suggests an encoding of convex-constraint programs as monotone-constraint programs. To present it, we need more notation. For a constraint $A=(X, C)$, we call the constraint $(X, \bar{C})$, where $\bar{C}=\mathcal{P}(X) \backslash C$, the dual constraint for $A$. We denote it by $\bar{A}$. It is a direct consequence of the definitions that a constraint $A$ is monotone if and only if its dual $\bar{A}$ is antimonotone.

Let $C$ be a convex constraint. We set $m c(C)=\{C\}$ if $C$ is monotone. We set $m c(C)=$ $\{\operatorname{not}(\bar{C})\}$, if $C$ is antimonotone. We define $m c(C)=\left\{C^{+}, \operatorname{not}\left(\overline{C^{-}}\right)\right\}$, if $C$ is neither monotone nor antimonotone. Clearly, $C$ and $m c(C)$ have the same models.

Let $P$ be a convex-constraint program. By $m c(P)$ we denote the program with monotone constraints obtained by replacing every rule $r$ in $P$ with a rule $r^{\prime}$ such that

$$
h d\left(r^{\prime}\right)=h d(r)^{+} \text {and } b d\left(r^{\prime}\right)=\bigcup\{m c(A): A \in b d(r)\}
$$

and, if $h d(r)$ is not monotone, also with an additional rule $r^{\prime \prime}$ such that

$$
h d\left(r^{\prime \prime}\right)=(\emptyset, \emptyset) \text { and } b d\left(r^{\prime \prime}\right)=\left\{\overline{h d(r)^{-}}\right\} \cup b d\left(r^{\prime}\right) .
$$

By our observation above, all constraints appearing in rules of $m c(P)$ are indeed monotone, that is, $m c(P)$ is a program with monotone constraints.

It follows from Proposition 8 that $M$ is a model of $P$ if and only if $M$ is a model of $m c(P)$. We extend this correspondence to supported and stable models of a convex constraint program $P$ and the monotone-constraint program $m c(P)$.

Definition 6. Let $P$ be a convex constraint program. Then a set of atoms $M$ is a supported (or stable) model of $P$ if $M$ is a supported (or stable) model of $m c(P)$.

With these definitions, monotone-constraint programs can be viewed (almost) directly as convexconstraint programs. Namely, we note that monotone and antimonotone constraints are convex. Next, we observe that if $A$ is a monotone constraint, the expression $\operatorname{not}(A)$ has the same meaning as the antimonotone constraint $\bar{A}$ in the sense that for every interpretation $M, M \models \operatorname{not}(A)$ if and only if $M \models \bar{A}$.

Let $P$ be a monotone-constraint program. By $c c(P)$ we denote the program obtained from $P$ by replacing every rule $r$ of the form (1) in $P$ with $r^{\prime}$ such that

$$
h d\left(r^{\prime}\right)=h d(r) \text { and } b d\left(r^{\prime}\right)=\bigcup\left\{A_{i}: i=1, \ldots, k\right\} \cup \bigcup\left\{\overline{A_{j}}: j=k+1, \ldots, m\right\}
$$

One can show that programs $P$ and $c c(P)$ have the same models, supported models and stable models. In fact, for every monotone-constraint program $P$ we have $P=m c(c c(P))$.

Remark. Another consequence of our discussion is that the default negation operator can be eliminated from the syntax at the price of allowing antimonotone constraints and using antimonotone constraints as negated literals.

Due to the correspondences we have established above, one can extend to convex-constraint programs all concepts and results we discussed earlier in the context of monotone-constraint programs. In many cases, they can also be stated directly in the language of convex-constraints. The 
most important for us are the notions of the completion and loop formulas, as they lead to new algorithms for computing stable models of lparse programs. Therefore, we will now discuss them in some detail.

As we just mentioned, we could use $\operatorname{Comp}(m c(P))$ as a definition of the completion $\operatorname{Comp}(P)$ for a convex-constraint logic program $P$. Under this definition Theorems 9 extends to the case of convex-constraint programs. However, $\operatorname{Comp}(m c(P))$ involves monotone constraints and their negations and not convex constraints that appear in $P$. Therefore, we will now propose another approach, which preserves convex constraints of $P$.

To this end, we first extend the logic $P L^{m c}$ with convex constraints. In this extension, which we denote by $P L^{c c}$ and refer to as the propositional logic with convex-constraints, formulas are boolean combinations of convex constraints. The semantics of such formulas is given by the notion of a model obtained by extending over boolean connectives the concept of a model of a convex constraint.

Thus, the only difference between the logic $P L^{m c}$, which we used to define the completion and loop completion for monotone-convex programs and the logic $P L^{c c}$ is that the former uses monotone constraints as building blocks of formulas, whereas the latter is based on convex constraints. In fact, since monotone constraints are special convex constraints, the logic $P L^{m c}$ is a fragment of the $\operatorname{logic} P L^{c c}$.

Let $P$ be a convex-constraint program. The completion of $P$, denoted by $\operatorname{Comp}(P)$, is the following set of $P L^{c c}$ formulas:

1. For every rule $r \in P$ we include in $\operatorname{Comp}(P)$ a $P L^{c c}$ formula

$$
[b d(r)]^{\wedge} \rightarrow h d(r)
$$

(as before, for a set of convex constraints $L, L^{\wedge}$ denotes the conjunction of the constraints in $L)$

2. For every atom $x \in A t(P)$, we include in $\operatorname{Comp}(P)$ a $P L^{c c}$ formula

$$
x \rightarrow \bigvee\left\{[b d(r)]^{\wedge}: r \in P, x \in h \operatorname{set}(r)\right\}
$$

(again, we note that when the set of rules in $P$ is infinite, the disjunction may be infinitary).

One can now show the following theorem.

Theorem 8. Let $P$ be a convex-constraint program and let $M \subseteq A t(P)$. Then $M$ is a supported model of $P$ if and only if $M$ is a model of $\operatorname{Comp}(P)$.

Proof. (Sketch) By the definition, $M$ is a supported model of $P$ if and only if $M$ is a supported model of $m c(P)$. It is a matter of routine checking that $\operatorname{Comp}(m c(P))$ and $\operatorname{Comp}(P)$ have the same models. Thus the assertion follows from Theorem 4 .

Next, we restrict attention to finitary convex-constraint programs, that is, programs with finite set of atoms, and extend to this class of programs the notions of the positive dependency graph and loops. To this end, we exploit its representation as a monotone-constraint program $m c(P)$. That is, we define the positive dependency graph, loops and loop formulas for $P$ as the positive dependency graph, loops and loop formulas of $m c(P)$, respectively. In particular, $L$ is a loop of $P$ if and only if 
$L$ is a loop of $m c(P)$ and the loop formula for $L$, with respect to a convex-constraint program $P$, is defined as the loop formula $L P(L)$ with respect to the program $m c(P)^{4}$. We note that since loop formulas for monotone-constraint programs only modify non-negated literals in the bodies of rules and leave negated literals intact, there seems to be no simple way to extend the notion of a loop formula to the case of a convex-constraint program $P$ without making references to $m c(P)$.

We now define a loop completion of a finitary convex-constraint program $P$ as the $P L^{c c}$ theory

$$
L \operatorname{Comp}(P)=\operatorname{Comp}(P) \cup\{L P(L): L \text { is a loop of } P\} .
$$

We have the following theorem that provides a necessary and sufficient condition for a set of atoms to be a stable model of a convex-constraint program.

Theorem 9. Let $P$ be a finitary convex-constraint program. A set $M \subseteq A t(P)$ is a stable model of $P$ if and only if $M$ is a model of $L C o m p(P)$.

Proof. (Sketch) Since $M$ is a stable model of $P$ if and only of $M$ is a stable model of $m c(P)$, Theorem 6 implies that $M$ is a stable model of $P$ if and only if $M$ is a stable model of $L \operatorname{Comp}(m c(P))$. It is a matter of routine checking that $\operatorname{LComp}(m c(P))$ and $L \operatorname{Comp}(P)$ have the same models. Thus, the result follows.

In a similar way, Theorem 7 implies the following result for convex-constraint programs.

Theorem 10. Let $P$ be a finitary convex-constraint program and $M$ a model of $C o m p(P)$. If $M^{-}$ is not empty, then $M$ violates the loop formula of every terminating loop of $G_{P}\left[M^{-}\right]$.

We emphasize that one could simply use $L \operatorname{Comp}(m c(P))$ as a definition of the loop completion for a convex-constraint logic program. However, our definition of the completion component of the loop completion retains the structure of constraints in a program $P$, which is important when using loop completion for computation of stable models, the topic we address in the next section of the paper.

\section{Applications}

In this section, we will use theoretical results on the program completion, loop formulas and loop completion of programs with convex constraints to design and implement a new method for computing stable models of lparse programs (Simons et al., 2002).

\subsection{Lparse Programs}

Simons et al. (2002) introduced and studied an extension of normal logic programming with weight atoms. Formally, a weight atom is an expression

$$
A=l\left[a_{1}=w_{1}, \ldots, a_{k}=w_{k}\right] u,
$$

where $a_{i}, 1 \leq i \leq k$ are propositional atoms, and $l, u$ and $w_{i}, 1 \leq i \leq k$ are non-negative integers. If all weights $w_{i}$ are equal to $1, A$ is a cardinality atom, written as $l\left\{a_{1}, \ldots, a_{k}\right\} u$.

4. There is one minor simplifi cation one might employ. For a monotone constraint $A, \neg A$ and $\bar{A}$ are equivalent and $\bar{A}$ is antimonotone and so, convex. Thus, we can eliminate the operator $\neg$ from loop formulas of convex-constraint programs by writing $\bar{A}$ instead of $\neg A$. 
An lparse rule is an expression of the form

$$
A \leftarrow A_{1}, \ldots, A_{n}
$$

where $A, A_{1}, \ldots, A_{n}$ are weight atoms. We refer to sets of lparse rules as lparse programs. Simons et al. (2002) defined for lparse programs the semantics of stable models.

A set $M$ of atoms is a model of (or satisfies) a weight atom $l\left[a_{1}=w_{1}, \ldots, a_{k}=w_{k}\right] u$ if

$$
l \leq \sum_{i=1}^{k}\left\{w_{i}: a_{i} \in M\right\} \leq u .
$$

With this semantics a weight atom $l\left[a_{1}=w_{1}, \ldots, a_{k}=w_{k}\right] u$ can be identified with a constraint $(X, C)$, where $X=\left\{a_{1}, \ldots, a_{k}\right\}$ and

$$
C=\left\{Y \subseteq X: l \leq \sum_{i=1}^{k}\left\{w_{i}: a_{i} \in Y\right\} \leq u\right\} .
$$

We notice that all weights in a weight atom $W$ are non-negative. Therefore, if $M \subseteq M^{\prime} \subseteq M^{\prime \prime}$ and both $M$ and $M^{\prime \prime}$ are models of $W$, then $M^{\prime}$ is also a model of $W$. It follows that the constraint $(X, C)$ we define above is convex.

Since $(X, C)$ is convex, weight atoms represent a class of convex constraints and lparse programs syntactically are a class of programs with convex constraints. This relationship extends to the stable-model semantics. Namely, Marek and Truszczyński (2004) and Marek et al. $(2004,2006)$ showed that lparse programs can be encoded as programs with monotone constraints so that the concept of a stable model is preserved. The transformation used there coincides with the encoding $m c$ described in the previous section, when we restrict the latter to lparse programs. Thus, we have the following theorem.

Theorem 11. Let $P$ be an lparse program. A set $M \subseteq$ At is a stable model of $P$ according to the definition by Simons et al. (2002) if and only if $M$ is a stable model of $P$ according to the definition given in the previous section (when $P$ is viewed as a convex-constraint program).

It follows that to compute stable models of lparse programs we can use the results obtained earlier in the paper, specifically the results on program completion and loop formulas for convexconstraint programs.

Remark. To be precise, the syntax of lparse programs is more general. It allows both atoms and negated atoms to appear within weight atoms. It also allows weights to be negative. However, negative weights in lparse programs are treated just as a notational convenience. Specifically, an expression of the form $a=w$ within a weight atom (where $w<0$ ) represents the expression $\operatorname{not}(a)=-w$ (eliminating negative weights in this way from a weight atom requires modifications of the bounds associated with this weight atom). Moreover, by introducing new propositional variables one can remove occurrences of negative literals from programs. These transformations preserve stable models (modulo new atoms). Marek and Truszczyński (2004) and Marek et al. (2004, 2006) provide a detailed discussion of this transformation.

In addition to weight atoms, the bodies of lparse rules may contain propositional literals (atoms and negated atoms) as conjuncts. We can replace these propositional literals with weight atoms 
as follows: an atom $a$ can be replaced with the cardinality atom $1\{a\}$, and a literal $\operatorname{not}(a)-$ with the cardinality atom $\{a\} 0$. This transformation preserves stable models, too. Moreover, the size of the resulting program does not increase more than by a constant factor. Thus, through the transformations discussed here, monotone- and convex-constraint programs capture arbitrary lparse programs.

\subsection{Computing Stable Models of Lparse Programs}

In this section we present an algorithm for computing stable models of lparse programs. Our method uses the results we obtained in Section 7 to reduce the problem to that of computing models of the loop completion of an lparse program. The loop completion is a formula in the logic $P L^{c c}$, in which the class of convex constraints is restricted to weight constraints, as defined in the previous subsection. We will denote the fragment of the logic $P L^{c c}$ consisting of such formulas by $P L^{w a}$.

To make the method practical, we need programs to compute models of theories in the logic $P L^{w a}$. We will now show a general way to adapt to this task off-the-shelf pseudo-boolean constraint solvers (Eén \& Sörensson, 2003; Aloul et al., 2002; Walser, 1997; Manquinho \& Roussel, 2005; Liu \& Truszczyński, 2003).

Pseudo-boolean constraints ( $P B$ for short) are integer programming constraints in which variables have 0-1 domains. We will write them as inequalities

$$
w_{1} \times x_{1}+\ldots+w_{k} \times x_{k} \operatorname{comp} w
$$

where comp stands for one of the relations $\leq, \geq,<$ and $>$, $w_{i}$ 's and $w$ are integer coefficients (not necessarily non-negative), and $x_{i}$ 's are integers taking value 0 or 1 . A set of pseudo-boolean constraints is a pseudo-boolean theory.

Pseudo-boolean constraints can be viewed as constraints. The basic idea is to treat each 0-1 variable $x$ as a propositional atom (which we will denote by the same letter). Under this correspondence, a pseudo-boolean constraint (3) is equivalent to the constraint $(X, C)$, where $X=\left\{x_{1}, \ldots, x_{k}\right\}$ and

$$
C=\left\{Y \subseteq X: \sum_{i=1}^{k}\left\{w_{i}: x_{i} \in Y\right\} \operatorname{comp} w\right\}
$$

in the sense that solutions to (3) correspond to models of $(X, C)\left(x_{i}=1\right.$ in a solution if and only if $x_{i}$ is true in the corresponding model). In particular, if all coefficients $w_{i}$ and the bound $w$ in (3) are non-negative, and if comp = ' $\geq$ ', then the constraint (3) is equivalent to a monotone lower-bound weight atom $w\left[x_{1}=w_{1}, \ldots, x_{n}=w_{n}\right]$.

It follows that an arbitrary weight atom can be represented by one or two pseudo-boolean constraints. More generally, an arbitrary $P L^{w a}$ formula $F$ can be encoded as a set of $P B$ constraints. We will describe the translation as a two-step process.

The first step consists of converting $F$ to a clausal form $\tau_{c l}(F)^{5}$. To control the size of the translation, we introduce auxiliary propositional atoms. Below, we describe the translation $F \mapsto$ $\tau_{c l}(F)$ under the assumption that $F$ is a formula of the loop completion of an lparse program $P$. Our main motivation is to compute stable models of logic programs and to this end algorithms for computing models of loop completions are sufficient.

5. A $P L^{w a}$ clause is any formula $B_{1} \wedge \ldots \wedge B_{m} \rightarrow H_{1} \vee \ldots \vee H_{n}$, where $B_{i}$ and $H_{j}$ are weight atoms. 
Let $F$ be a formula in the loop completion of an lparse-program $P$. We define $\tau_{c l}(F)$ as follows (in the transformation, we use a propositional atom $x$ as a shorthand for the cardinality atom $C(x)=$ $1\{x\})$.

1. If $F$ is of the form $A_{1} \wedge \ldots \wedge A_{n} \rightarrow A$, then $\tau_{c l}(F)=F$

2. If $F$ is of the form $x \rightarrow\left(\left[b d\left(r_{1}\right)\right]^{\wedge}\right) \vee \ldots \vee\left(\left[b d\left(r_{l}\right)\right]^{\wedge}\right)$, then we introduce new propositional atoms $b_{r, 1}, \ldots, b_{r, l}$ and set $\tau_{c l}(F)$ to consist of the following $P L^{w a}$ clauses:

$$
\begin{gathered}
x \rightarrow b_{r, 1} \vee \ldots \vee b_{r, l} \\
{\left[b d\left(r_{i}\right)\right]^{\wedge} \rightarrow b_{r, i}, \text { for every } b d\left(r_{i}\right)} \\
b_{r, i} \rightarrow A_{j}, \text { for every } b d\left(r_{i}\right) \text { and } A_{j} \in b d\left(r_{i}\right)
\end{gathered}
$$

3. If $F$ is of the form $\bigvee L \rightarrow \bigvee_{r}\left\{\beta_{L}(r)\right\}$, where $L$ is a set of atoms, and every $\beta_{L}(r)$ is a conjunction of weight atoms, then we introduce new propositional atoms $b d f_{L, r}$ for every $\beta_{L}(r)$ in $F$ and represent $\bigvee L$ as the weight atom $W_{L}=1\left[l_{i}=1: l_{i} \in L\right]$. We then define $\tau_{c l}(F)$ to consist of the following clauses:

$$
\begin{gathered}
W_{L} \rightarrow \bigvee b d f_{L, r} \\
\beta_{L}(r) \rightarrow b d f_{L, r}, \text { for every } \beta_{L}(r) \in F \\
b d f_{L, r} \rightarrow A_{j}, \text { for every } \beta_{L}(r) \in F \text { and } A_{j} \in \beta_{L}(r) .
\end{gathered}
$$

It is clear that the size $\tau_{c l}(F)$ is linear in the size of $F$.

The second step of the translation, converts a $P L^{w a}$ formula $C$ in a clausal form into a set of $P B$ constraints, $\tau_{p b}(C)$. To define the translation $C \rightarrow \tau_{p b}(C)$, let us consider a $P L^{w a}$ clause $C$ of the form

$$
B_{1} \wedge \ldots \wedge B_{m} \rightarrow H_{1} \vee \ldots \vee H_{n}
$$

where $B_{i}$ 's and $H_{i}$ 's are weight atoms.

We introduce new propositional atoms $b_{1}, \ldots, b_{m}$ and $h_{1}, \ldots, h_{n}$ to represent each weight atom in the clause. As noted earlier in the paper, we simply write $x$ for a weight atoms of the form $1[x=$ $1]$. With the new atoms, the clause (4) becomes a propositional clause $b_{1} \wedge \ldots \wedge b_{m} \rightarrow h_{1} \vee \ldots \vee h_{n}$. We represent it by the following $P B$ constraint:

$$
-b_{1}-\ldots-b_{m}+h_{1}+\ldots+h_{n} \geq 1-m .
$$

Here and later in the paper, we use the same symbols to denote propositional variables and the corresponding 0-1 integer variables. The context will always imply the correct meaning of the symbols. Under this convention, it is easy to see that a propositional clause $b_{1} \wedge \ldots \wedge b_{m} \rightarrow h_{1} \vee \ldots \vee h_{n}$ and its $P B$ constraint (5) have the same models.

We introduce next $P B$ constraints that enforce the equivalence of the newly introduced atoms $b_{i}$ (or $h_{i}$ ) and the corresponding weight atoms $B_{i}$ (or $H_{i}$ ).

Let $B=l\left[a_{1}=w_{1}, \ldots, a_{k}=w_{k}\right] u$ be a weight atom and $b$ a propositional atom. We split $B$ to $B^{+}$and $B^{-}$and introduce two more atoms $b^{+}$and $b^{-}$. To model $B \equiv b$, we model with pseudoboolean constraints the following three equivalences: $b \equiv b^{+} \wedge b^{-}, b^{+} \equiv B^{+}$, and $b^{-} \equiv B^{-}$.

1. The first equivalence can be captured with three propositional clauses. Hence the following three $P B$ constraints model that equivalence:

$$
-b+b^{+} \geq 0
$$




$$
\begin{gathered}
-b+b^{-} \geq 0 \\
-b^{+}-b^{-}+b \geq-1
\end{gathered}
$$

2. The second equivalence, $b^{+} \equiv B^{+}$, can be modeled by the following two $P B$ constraints

$$
\begin{gathered}
(-l) \times b^{+}+\sum_{i=1}^{k}\left(a_{i} \times w_{i}\right) \geq 0 \\
-\left(\sum_{i=1}^{k} w_{i}-l+1\right) \times b^{+}+\sum_{i=1}^{k}\left(a_{i} \times w_{i}\right) \leq l-1
\end{gathered}
$$

3. Similarly, the third equivalence, $b^{-} \equiv B^{-}$, can be modeled by the following two $P B$ constraints

$$
\begin{gathered}
\left(\sum_{i=1}^{k} w_{i}-u\right) \times b^{-}+\sum_{i=1}^{k}\left(a_{i} \times w_{i}\right) \leq \sum_{i=1}^{k} w_{i} \\
(u+1) \times b^{-}+\sum_{i=1}^{k}\left(a_{i} \times w_{i}\right) \geq u+1
\end{gathered}
$$

We define now $\tau_{p b}(C)$, for a $P L^{w a}$ clause $C$, as the set of all pseudo-boolean constraints (5) and (6), (7), (8), (11), (12), (9), (10) constructed for every weight atom occurring in $C$. One can verify that the size of $\tau_{p b}(C)$ is linear in the size of $C$. Therefore, $\tau_{p b}\left(\tau_{c l}(F)\right)$ has size linear in the size of $F$.

In the special case where all $B_{i}$ 's and $H_{j}$ 's are weight atoms of the form $1\left[b_{i}=1\right]$ and $1\left[h_{j}=1\right]$, we do not need to introduce any new atoms and $P B$ constraints (6), (7), (8), (11), (12), (9), (10). Then $\tau_{p b}(C)$ consists of a single $P B$ constraint (5).

We have the following theorem establishing the correctness of the transformation $\tau$. The proof of the theorem is straightforward.

Theorem 12. Let $F$ be a loop completion formula in logic $P L^{w a}$, and $M$ a set of atoms, $M \subseteq$ At $(F)$. Then $M$ is a model of $F$ in $P L^{w a}$ logic if and only if $M$ has a unique extension $M^{\prime}$ by some of the new atoms in $A t\left(\tau_{p b}\left(\tau_{c l}(F)\right)\right)$ such that $M^{\prime}$ is a model of the pseudo-boolean theory $\tau_{p b}\left(\tau_{c l}(F)\right)$.

We note that when we use solvers designed for $P L^{w a}$ theories, then translation $\tau_{p b}$ is no longer needed. The benefit of using such solvers is that we do not need to split weight atoms in the $P L^{w a}$ theories and do not need the auxiliary atoms introduced in $\tau_{p b}$.

\subsubsection{THE Algorithm}

We follow the approach proposed by Lin and Zhao (2002). As in that paper, we first compute the completion of a lparse program. Then, we iteratively compute models of the completion using a $P B$ solver. Whenever a model is found, we test it for stability. If the model is not a stable model of the program, we extend the completion by loop formulas identified in Theorem 10. Often, adding a single loop formula filters out several models of $\operatorname{Comp}(P)$ that are not stable models of $P$.

The results given in the previous section ensure that our algorithm is correct. We present it in Figure 1 . We note that it may happen that in the worst case exponentially many loop formulas are 


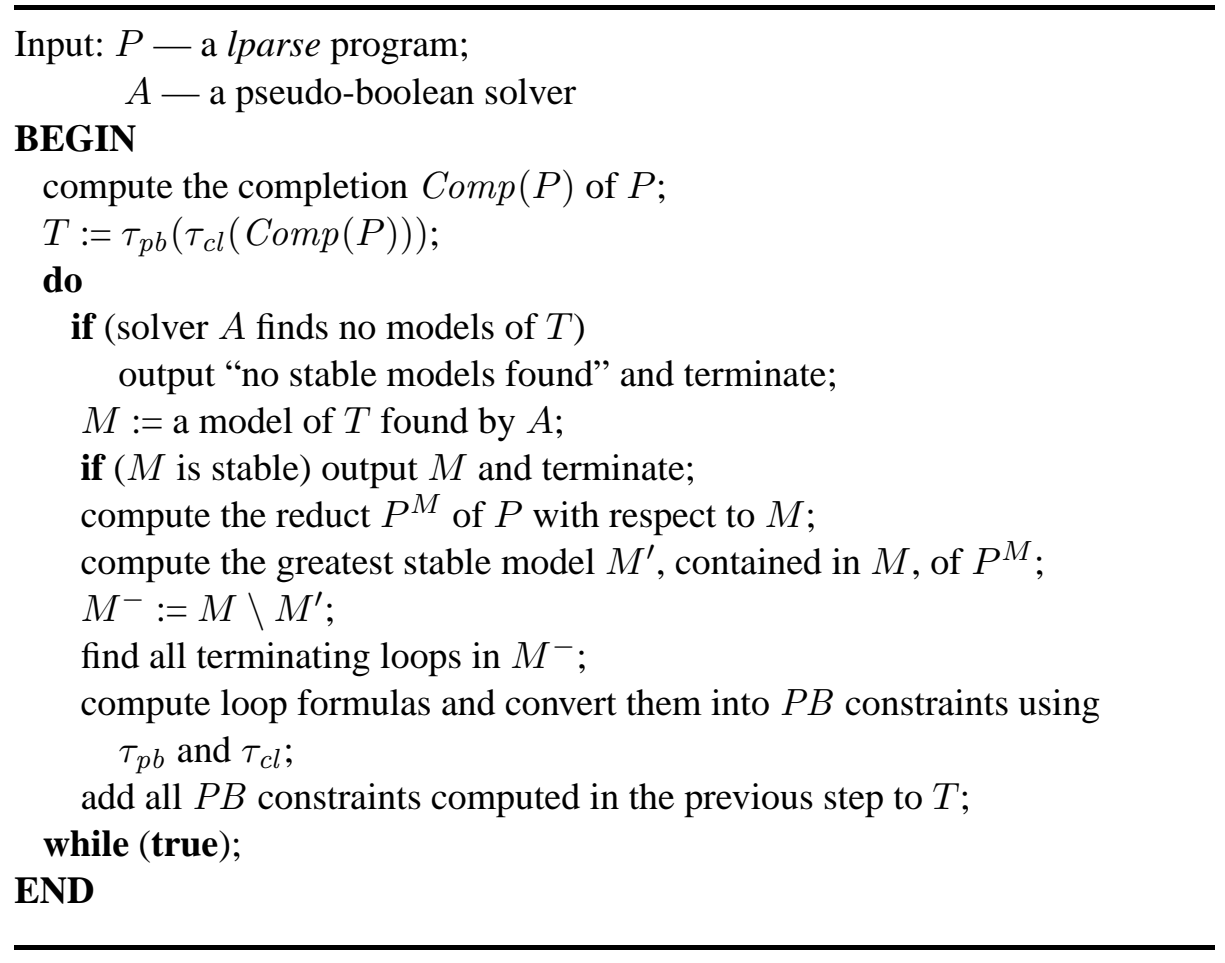

Figure 1: Algorithm of pbmodels

needed before the first stable model is found or we determine that no stable models exist (Lin \& Zhao, 2002). However, that problem arises only rarely in practical situations ${ }^{6}$.

The implementation of pbmodels supports several $P B$ solvers such as satzoo (Eén \& Sörensson, 2003), pbs (Aloul et al., 2002), wsatoip (Walser, 1997). It also supports a program wsatcc (Liu \& Truszczyński, 2003) for computing models of $P L^{w a}$ theories. When this last program is used, the transformation, from "clausal" $P L^{w a}$ theories to pseudo-boolean theories is not needed. The first two of these four programs are complete $P B$ solvers. The latter two are local-search solvers based on wsat (Selman, Kautz, \& Cohen, 1994).

We output the message "no stable model found" in the first line of the loop and not simply "no stable models exist" since in the case when $A$ is a local-search algorithm, failure to find a model of the completion (extended with loop formulas in iteration two and the subsequent ones) does not imply that no models exist.

\subsection{Performance}

In this section, we present experimental results concerning the performance of pbmodels. The experiments compared pbmodels, combined with several $P B$ solvers, to smodels (Simons et al., 2002) and cmodels (Babovich \& Lifschitz, 2002). We focused our experiments on problems whose state-

6. In fact, in many cases programs turn out to be tight with respect to their supported models. Therefore, supported models are stable and no loop formulas are necessary at all. 
ments explicitly involve pseudo-boolean constraints, as we designed pbmodels with such problems in mind.

For most benchmark problems we tried cmodels did not perform well. Only in one case (vertexcover benchmark) the performance of cmodels was competitive, although even in this case it was not the best performer. Therefore, we do not report here results we compiled for cmodels. For a complete set of results we obtained in the experiments we refer to http://www.cs.uky .edu/ ai/pbmodels.

In the experiments we used instances of the following problems: traveling salesperson, weighted $n$-queens, weighted Latin square, magic square, vertex cover, and Towers of Hanoi. The lparse programs we used for the first four problems involve general pseudo-boolean constraints. Programs modeling the last two problems contain cardinality constraints only.

Traveling salesperson problem (TSP). An instance consists of a weighted complete graph with $n$ vertices, and a bound $w$. All edge weights and $w$ are non-negative integers. A solution to an instance is a Hamiltonian cycle whose total weight (the sum of the weights of all its edges) is less than or equal to $w$.

We randomly generated 50 weighted complete graphs with 20 vertices, To this end, in each case we assign to every edge of a complete undirected graph an integer weight selected uniformly at random from the range [1..19]. By setting $w$ to 100 we obtained a set of "easy" instances, denoted by TSP-e (the bound is high enough for every instance in the set to have a solution). From the same collection of graphs, we also created a set of "hard" instances, denoted by TSP- $h$, by setting $w$ to 62 . Since the requirement on the total weight is stronger, the instances in this set in general take more time.

Weighted $n$-queens problem (WNQ). An instance to the problem consists of a weighted $n \times n$ chess board and a bound $w$. All weights and the bound are non-negative integers. A solution to an instance is a placement of $n$ queens on the chess board so that no two queens attack each other and the weight of the placement (the sum of the weights of the squares with queens) is not greater than $w$.

We randomly generated 50 weighted chess boards of the size $20 \times 20$, where each chess board is represented by a set of $n \times n$ integer weights $w_{i, j}, 1 \leq i, j \leq n$, all selected uniformly at random from the range [1..19]. We then created two sets of instances, easy (denoted by wnq-e) and hard (denoted by $w n q-h$ ), by setting the bound $w$ to 70 and 50, respectively.

Weighted Latin square problem (WLSQ). An instance consists of an $n \times n$ array of weights $w_{i, j}$, and a bound $w$. All weights $w_{i, j}$ and $w$ are non-negative integers. A solution to an instance is an $n \times n$ array $L$ with all entries from $\{1, \ldots, n\}$ and such that each element in $\{1, \ldots, n\}$ occurs exactly once in each row and in each column of $L$, and $\sum_{i=1}^{n} \sum_{j=1}^{n} L[i, j] \times w_{i, j} \leq w$.

We set $n=10$ and we randomly generated 50 sets of integer weights, selecting them uniformly at random from the range [1..9]. Again we created two families of instances, easy (wlsq-e) and hard ( $w l s q-h$ ), by setting $w$ to 280 and 225 , respectively.

Magic square problem. An instance consists of a positive integer $n$. The goal is to construct an $n \times n$ array using each integer $1, \ldots n^{2}$ as an entry in the array exactly once in such a way that entries in each row, each column and in both main diagonals sum up to $n\left(n^{2}+1\right) / 2$. For the experiments we used the magic square problem for $n=4,5$ and 6 .

Vertex cover problem. An instance consists of graph with $n$ vertices and $m$ edges, and a nonnegative integer $k-$ a bound. A solution to the instance is a subset of vertices of the graph with no more than $k$ vertices and such that at least one end vertex of every edge in the graph is in the subset. 
We randomly generated 50 graphs, each with 80 vertices and 400 edges. For each graph, we set $k$ to be a smallest integer such that a vertex cover with that many elements still exists.

Towers of Hanoi problem. This is a slight generalization of the original problem. We considered the case with six disks and three pegs. An instance consists of an initial configuration of disks that satisfies the constraint of the problem (larger disk must not be on top of a smaller one) but does not necessarily requires that all disks are on one peg. These initial configurations were selected so that they were 31,36, 41 and 63 steps away from the goal configuration (all disks from the largest to the smallest on the third peg), respectively. We also considered a standard version of the problem with seven disks, in which the initial configuration is 127 steps away from the goal.

We encoded each of these problems as a program in the general syntax of lparse, which allows the use of relation symbols and variables (Syrjänen, 1999). The programs are available at http: //www.cs.uky.edu/ai/pbmodels. We then used these programs in combination with appropriate instances as inputs to lparse (Syrjänen, 1999). In this way, for each problem and each set of instances we generated a family of ground (propositional) lparse programs so that stable models of each of these programs represent solutions to the corresponding instances of the problem (if there are no stable models, there are no solutions). We used these families of lparse programs as inputs to solvers we were testing. All these ground programs are also available at http: //www.cs.uky.edu/ai/pbmodels.

In the tests, we used pbmodels with the following four $P B$ solvers: satzoo (Eén \& Sörensson, 2003), pbs (Aloul et al., 2002), wsatcc (Liu \& Truszczyński, 2003), and wsatoip (Walser, 1997). In particular, wsatcc deals with $P L^{w a}$ theories directly.

All experiments were run on machines with $3.2 \mathrm{GHz}$ Pentium 4 CPU, 1GB memory, running Linux with kernel version 2.6.11, gec version 3.3.4. In all cases, we used 1000 seconds as the timeout limit.

We first show the results for the magic square and towers of Hanoi problems. In Table 1, for each solver and each instance, we report the corresponding running time in seconds. Local-search solvers were unable to solve any of the instances in the two problems and so are not included in the table.

\begin{tabular}{|c|c|c|c|}
\hline Benchmark & smodels & pbmodels-satzoo & pbmodels-pbs \\
\hline \hline magic square $(4 \times 4)$ & 1.36 & 1.70 & 2.41 \\
\hline magic square $(5 \times 5)$ & $>1000$ & 28.13 & 0.31 \\
\hline magic square $(6 \times 6)$ & $>1000$ & 75.58 & $>1000$ \\
\hline towers of Hanoi $(d=6, t=31)$ & 16.19 & 18.47 & 1.44 \\
\hline towers of Hanoi $(d=6, t=36)$ & 32.21 & 31.72 & 1.54 \\
\hline towers of Hanoi $(d=6, t=41)$ & 296.32 & 49.90 & 3.12 \\
\hline towers of Hanoi $(d=6, t=63)$ & $>1000$ & $>1000$ & 3.67 \\
\hline towers of Hanoi $(d=7, t=127)$ & $>1000$ & $>1000$ & 22.83 \\
\hline
\end{tabular}

Table 1: Magic square and towers of Hanoi problems

Both pbmodels-satzoo and pbmodels-pbs perform better than smodels on programs obtained from the instances of both problems. We observe that pbmodels-pbs performs exceptionally well in the tower of Hanoi problem. It is the only solver that can compute a plan for 7 disks, which requires 127 steps. Magic square and Towers of Hanoi problems are highly regular. Such problems 


\begin{tabular}{|c|c|c|c|}
\hline & \# of SAT instances & \# of UNSAT instances & \# of UNKNOWN instances \\
\hline \hline TSP-e & 50 & 0 & 0 \\
\hline TSP- $h$ & 31 & 1 & 18 \\
\hline$w n q-e$ & 49 & 0 & 1 \\
\hline$w n q-h$ & 29 & 0 & 21 \\
\hline wlsq-e & 45 & 4 & 1 \\
\hline$w l s q-h$ & 8 & 41 & 1 \\
\hline vtxcov & 50 & 0 & 0 \\
\hline
\end{tabular}

Table 2: Summary of Instances

\begin{tabular}{|c|c|c|c|}
\hline & smodels & pbmodels-satzoo & pbmodels-pbs \\
\hline \hline TSP-e & $45 / 17$ & $50 / 30$ & $18 / 3$ \\
\hline TSP- $h$ & $7 / 3$ & $16 / 14$ & $0 / 0$ \\
\hline wnq-e & $11 / 5$ & $26 / 23$ & $0 / 0$ \\
\hline wnq- $h$ & $2 / 2$ & $0 / 0$ & $0 / 0$ \\
\hline wlsq-e & $21 / 1$ & $49 / 29$ & $46 / 19$ \\
\hline wlsq- $h$ & $0 / 0$ & $47 / 26$ & $47 / 23$ \\
\hline vtxcov & $50 / 40$ & $50 / 1$ & $47 / 3$ \\
\hline sum over all & $136 / 68$ & $238 / 123$ & $158 / 48$ \\
\hline
\end{tabular}

Table 3: Summary on all instances

are often a challenge for local-search problems, which may explain a poor performance we observed for pbmodels-wsatcc and pbmodels-wsatoip on these two benchmarks.

For the remaining four problems, we used 50-element families of instances, which we generated randomly in the way discussed above. We studied the performance of complete solvers (smodels, pbmodels-satzoo and pbmodels-pbs) on all instances. We then included local-search solvers (pbmodels-wsatcc and pbmodelswsatoip) in the comparisons but restricted attention only to instances that were determined to be satisfiable (as local-search solvers are, by their design, unable to decide unsatisfiability). In Table 2, for each family we list how many of its instances are satisfiable, unsatisfiable, and for how many of the instances none of the solvers we tried was able to decide satisfiability.

In Table 3, for each of the seven families of instances and for each complete solver, we report two values $s / w$, where $s$ is the number of instances solved by the solver and $w$ is the number of times it was the fastest among the three.

The results in Table 3 show that overall pbmodels-satzoo solved more instances than pbmodels$p b s$, followed by smodels. When we look at the number of times a solver was the fastest one, pbmodels-satzoo was a clear winner overall, followed by smodels and then by pbmodels-pbs. Looking at the seven families of tests individually, we see that pbmodels-satzoo performed better than the other two solvers on five of the families. On the other two smodels was the best performer (although, it is a clear winner only on the vertex-cover benchmark; all solvers were essentially ineffective on the $w n q-h)$.

We also studied the performance of pbmodels combined with local-search solvers wsatcc (Liu \& Truszczyński, 2003) and wsatoip (Walser, 1997). For this study, we considered only those instances in the seven families that we knew were satisfiable. Table 4 presents results for all solvers 


\begin{tabular}{|c|c|c|c|c|c|}
\hline & smodels & pbmd-satzoo & pbmd-pbs & pbmd-wsatcc & pbmd-wsatoip \\
\hline \hline TSP-e & $45 / 3$ & $50 / 5$ & $18 / 2$ & $32 / 7$ & $47 / 34$ \\
\hline TSP- $h$ & $7 / 0$ & $16 / 2$ & $0 / 0$ & $19 / 6$ & $28 / 22$ \\
\hline wnq-e & $11 / 0$ & $26 / 0$ & $0 / 0$ & $49 / 45$ & $49 / 4$ \\
\hline wnq- $h$ & $2 / 0$ & $0 / 0$ & $0 / 0$ & $29 / 15$ & $29 / 14$ \\
\hline wlsq-e & $21 / 0$ & $45 / 0$ & $44 / 0$ & $45 / 33$ & $45 / 14$ \\
\hline wlsq- $h$ & $0 / 0$ & $7 / 0$ & $8 / 0$ & $7 / 1$ & $8 / 7$ \\
\hline vtxcov & $50 / 0$ & $50 / 0$ & $47 / 0$ & $50 / 36$ & $50 / 15$ \\
\hline sum over all & $136 / 3$ & $194 / 7$ & $117 / 2$ & $231 / 143$ & $256 / 110$ \\
\hline
\end{tabular}

Table 4: Summary on SAT instances

we studied (including the complete ones). As before, each entry provides a pair of numbers $s / w$, where $s$ is the number of solved instances and $w$ is the number of times the solver performed better than its competitors.

The results show superior performance of pbmodels combined with local-search solvers. They solve more instances than complete solvers (including smodels). In addition, they are significantly faster, winning much more frequently than complete solvers do (complete solvers were faster only on 12 instances, while local-search solvers were faster on 253 instances).

Our results demonstrate that pbmodels with solvers of pseudo-boolean constraints outperforms smodels on several types of search problems involving pseudo-boolean (weight) constraints).

We note that we also analyzed the run-time distributions for each of these families of instances. A run-time distribution is regarded as a more accurate and detailed measure of the performance of algorithms on randomly generated instances ${ }^{7}$. The results are consistent with the summary results presented above and confirm our conclusions. As the discussion of run-time distributions requires much space, we do not include this analysis here. They are available at the website http: / www . cs.uky.edu/ai/pbmodels.

\section{Related work}

Extensions of logic programming with means to model properties of sets (typically consisting of ground terms) have been extensively studied. Usually, these extensions are referred to by the common term of logic programming with aggregates. The term comes from the fact that most properties of sets of practical interest are defined through "aggregate" operations such as sum, count, maximum, minimum and average. We chose the term constraint to stress that we speak about abstract properties that define constraints on truth assignments (which we view as sets of atoms).

Mumick, Pirahesh, and Ramakrishnan (1990), and Kemp and Stuckey (1991) were among the first to study logic programs with aggregates. Recently, Niemelä et al. (1999) and Simons et al. (2002) introduced the class of lparse programs. We discussed this formalism in detail earlier in this paper.

Pelov (2004) and Pelov et al. (2006) studied a more general class of aggregates and developed a systematic theory of aggregates in logic programming based on the approximation theory (Denecker, Marek, \& Truszczyński, 2000). The resulting theory covers not only the stable models semantics but also the supported-model semantics and extensions of 3-valued Kripke-Kleene and

7. Hoos and Stützle (2005) provide a detailed discussion of this matter in the context of local-search methods. 
well-founded semantics. The formalism introduced and studied by Pelov (2004) and Pelov et al. (2006) allows for arbitrary aggregates (not only monotone ones) to appear in the bodies of rules. However, it does not allow for aggregates to appear in the heads of program clauses. Due to differences in the syntax and the scope of semantics studied there is no simple way to relate Pelov's (2004) and Pelov et al.'s (2006) formalism to programs with monotone (convex) constraints. We note though that programs with abstract monotone constraints with the heads of rules of the form $C(a)$ can be viewed almost literally as programs in the formalism by Pelov (2004) and Pelov et al. (2006) and that they have the same stable models according to the definitions we used in this paper and those by Pelov (2004) and Pelov et al. (2006).

Faber et al. (2004) developed the theory of disjunctive logic programs with aggregates. Similarly as Pelov (2004) and Pelov et al. (2006), Faber et al. (2004) do not allow for aggregates to appear in the heads of program clauses. This is one of the differences between that approach and programs with monotone (convex) constraints we studied here. The other major difference is related to the postulate of the minimality of stable models (called answer sets in the context of the formalism considered by Faber et al., 2004). In keeping with the spirit of the original answer-set semantics (Gelfond \& Lifschitz, 1991), answer sets of disjunctive programs with aggregates, as defined by Faber et al. (2004), are minimal models. Stable models of programs with abstract constraints do not have this property. However, for the class of programs with abstract monotone constraints with the heads of rules of the form $C(a)$ the semantics of answer sets defined by Faber et al. (2004) coincides with the semantics of stable models by Marek and Truszczyński (2004) and Marek et al. (2004, 2006).

Yet another approach to aggregates in logic programming was presented by Son and Pontelli (2006). That approach considered programs of the syntax similar to programs with monotone abstract constraints. It allowed arbitrary constraints (not only monotone ones) but not under the scope of not operator. A general principle behind the definition of the stable-model semantics by Son and Pontelli (2006) is to view a program with constraints as a concise representation of a set of its "instances", each being a normal logic program. Stable models of the program with constraints are defined as stable models of its instances and is quite different from the operator-based definition by Marek and Truszczyński (2004) and Marek et al. (2004, 2006). However, for programs with monotone constraint atoms which fall in the scope of the formalism of Son and Pontelli (2006) both approaches coincide.

We also note that recently Son et al. (2006) presented a conservative extension of the syntax proposed by Marek and Truszczyński (2004) Marek et al. (2006), in which clauses are built of arbitrary constraint atoms.

Finally, we point out the work by Ferraris and Lifschitz (2004) and Ferraris (2005) which treats aggregates as nested expressions. In particular, Ferraris (2005) introduces a propositional logic with a certain nonclassical semantics, and shows that it extends several approaches to programs with aggregates, including those by Simons et al. (2002) (restricted to core lparse programs) and Faber et al. (2004). The nature of the relationship of the formalism by Ferraris (2005) and programs with abstract constraints remains an open problem.

\section{Conclusions}

Our work shows that concepts, techniques and results from normal logic programming, concerning strong and uniform equivalence, tightness and Fages lemma, program completion and loop formu- 
las, generalize to the abstract setting of programs with monotone and convex constraints. These general properties specialize to new results about lparse programs (with the exception of the characterization strong equivalence of lparse programs, which was first obtained by Turner, 2003).

Given these results we implemented a new software pbmodels for computing stable models of lparse programs. The approach reduces the problem to that of computing models of theories consisting of pseudo-boolean constraints, for which several fast solvers exist (Manquinho \& Roussel, 2005). Our experimental results show that pbmodels with $P B$ solvers, especially local search $P B$ solvers, performs better than smodels on several types of search problems we tested. Moreover, as new and more efficient solvers of pseudo-boolean constraints become available (the problem is receiving much attention in the satisfiability and integer programming communities), the performance of pbmodels will improve accordingly.

\section{Acknowledgments}

We acknowledge the support of NSF grants IIS-0097278 and IIS-0325063. We are grateful to the reviewers for their useful comments and suggestions.

This paper combines and extends results included in conference papers (Liu \& Truszczyński, 2005b, 2005a).

\section{References}

Aloul, F., Ramani, A., Markov, I., \& Sakallah, K. (2002). PBS: a backtrack-search pseudo-boolean solver and optimizer. In Proceedings of the 5th International Symposium on Theory and Applications of Satisfi ability, pp. $346-353$.

Babovich, Y., \& Lifschitz, V. (2002). Cmodels package.. http://www.cs.utexas.edu/users/ tag/cmodels.html.

Baral, C. (2003). Knowledge representation, reasoning and declarative problem solving. Cambridge University Press.

Clark, K. (1978). Negation as failure. In Gallaire, H., \& Minker, J. (Eds.), Logic and data bases, pp. 293-322. Plenum Press, New York-London.

Dell'Armi, T., Faber, W., Ielpa, G., Leone, N., \& Pfeifer, G. (2003). Aggregate functions in disjunctive logic programming: semantics, complexity, and implementation in DLV. In Proceedings of the 18th International Joint Conference on Artifi cial Intelligence (IJCAI-2003), pp. 847-852. Morgan Kaufmann.

Denecker, M., Marek, V., \& Truszczyński, M. (2000). Approximations, stable operators, well-founded fi xpoints and applications in nonmonotonic reasoning. In Minker, J. (Ed.), Logic-Based Artifi cial Intelligence, pp. 127-144. Kluwer Academic Publishers.

Eén, N., \& Sörensson, N. (2003). An extensible SAT solver. In Theory and Applications of Satisfi ability Testing, 6th International Conference, SAT-2003, Vol. 2919 of LNCS, pp. 502-518. Springer.

Eiter, T., \& Fink, M. (2003). Uniform equivalence of logic programs under the stable model semantics. In Proceedings of the 2003 International Conference on Logic Programming, Vol. 2916 of Lecture Notes in Computer Science, pp. 224-238, Berlin. Springer.

Erdem, E., \& Lifschitz, V. (2003). Tight logic programs. Theory and Practice of Logic Programming, 3(4-5), 499-518.

Faber, W., Leone, N., \& Pfeifer, G. (2004). Recursive aggregates in disjunctive logic programs: Semantics and complexity.. In Proceedings of the 9th European Conference on Artifi cial Intelligence (JELIA 2004), Vol. 3229 of LNAI, pp. 200 - 212. Springer. 
Fages, F. (1994). Consistency of Clark's completion and existence of stable models. Journal of Methods of Logic in Computer Science, 1, 51-60.

Ferraris, P. (2005). Answer sets for propositional theories. In Logic Programming and Nonmonotonic Reasoning, 8th International Conference, LPNMR 2005, Vol. 3662 of LNAI, pp. 119-131. Springer.

Ferraris, P., \& Lifschitz, V. (2004). Weight constraints ans nested expressions. Theory and Practice of Logic Programming, 5, 45-74.

Gelfond, M., \& Leone, N. (2002). Logic programming and knowledge representation - the A-prolog perspective. Artifi cial Intelligence, 138, 3-38.

Gelfond, M., \& Lifschitz, V. (1988). The stable semantics for logic programs. In Proceedings of the 5th International Conference on Logic Programming, pp. 1070-1080. MIT Press.

Gelfond, M., \& Lifschitz, V. (1991). Classical negation in logic programs and disjunctive databases. New Generation Computing, 9, 365-385.

Hoos, H., \& Stützle, T. (2005). Stochastic Local Search Algorithms — Foundations and Applications. Morgan-Kaufmann.

Kemp, D., \& Stuckey, P. (1991). Semantics of logic programs with aggregates. In Logic Programming, Proceedings of the 1991 International Symposium, pp. 387-401. MIT Press.

Lifschitz, V., Pearce, D., \& Valverde, A. (2001). Strongly equivalent logic programs. ACM Transactions on Computational Logic, 2(4), 526-541.

Lin, F. (2002). Reducing strong equivalence of logic programs to entailment in classical propositional logic. In Principles of Knowledge Representation and Reasoning, Proceedings of the 8th International Conference (KR2002). Morgan Kaufmann Publishers.

Lin, F., \& Zhao, Y. (2002). ASSAT: Computing answer sets of a logic program by SAT solvers. In Proceedings of the 18th National Conference on Artifi cial Intelligence (AAAI-2002), pp. 112-117. AAAI Press.

Liu, L., \& Truszczyński, M. (2003). Local-search techniques in propositional logic extended with cardinality atoms. In Rossi, F. (Ed.), Proceedings of the 9th International Conference on Principles and Practice of Constraint Programming, CP-2003, Vol. 2833 of LNCS, pp. 495-509. Springer.

Liu, L., \& Truszczyński, M. (2005a). Pbmodels - software to compute stable models by pseudoboolean solvers. In Logic Programming and Nonmonotonic Reasoning, Proceedings of the 8th International Conference (LPNMR-05), LNAI 3662, pp. 410-415. Springer.

Liu, L., \& Truszczyński, M. (2005b). Properties of programs with monotone and convex constraints. In Proceedings of the 20th National Conference on Artifi cial Intelligence (AAAI-05), pp. 701-706. AAAI Press.

Manquinho, V., \& Roussel, O. (2005). Pseudo boolean evaluation 2005.. http://www.cril. univ-artois.fr/PB05/.

Marek, V., Niemelä, I., \& Truszczyński, M. (2004). Characterizing stable models of logic programs with cardinality constraints. In Proceedings of the 7th International Conference on Logic Programming and Nonmonotonic Reasoning, Vol. 2923 of Lecture Notes in Artifi cial Intelligence, pp. 154-166. Springer.

Marek, V., Niemelä, I., \& Truszczyński, M. (2006). Logic programs with monotone abstract constraint atoms. Theory and Practice of Logic Programming. Submitted.

Marek, V., \& Truszczyński, M. (2004). Logic programs with abstract constraint atoms. In Proceedings of the 19th National Conference on Artifi cial Intelligence (AAAI-04), pp. 86-91. AAAI Press.

Mumick, I., Pirahesh, H., \& Ramakrishnan, R. (1990). The magic of duplicates and aggregates. In Proceedings of the 16th International Conference on Very Large Data Bases, VLDB 1990, pp. 264-277. Morgan Kaufmann. 
Niemelä, I., Simons, P., \& Soininen, T. (1999). Stable model semantics of weight constraint rules. In Proceedings of LPNMR-1999, Vol. 1730 of LNAI, pp. 317-331. Springer.

Pelov, N. (2004). Semantics of logic programs with aggregates. PhD Thesis. Department of Computer Science, K.U.Leuven, Leuven, Belgium.

Pelov, N., Denecker, M., \& Bruynooghe, M. (2004). Partial stable models for logic programs with aggregates. In Lifschitz, V., \& Niemelä, I. (Eds.), Logic programming and Nonmonotonic Reasoning, Proceedings of the $7^{\text {th }}$ International Conference, Vol. 2923, pp. 207-219. Springer.

Pelov, N., Denecker, M., \& Bruynooghe, M. (2006). Well-founded and stable semantics of logic programs with aggregates. Theory and Practice of Logic Programming. Accepted (available at http: //www . Cs.kuleuven.ac.be/〜dtai/projects/ALP/TPLP/).

Selman, B., Kautz, H., \& Cohen, B. (1994). Noise strategies for improving local search. In Proceedings of the 12th National Conference on Artifi cial Intelligence (AAAI-1994), pp. 337-343, Seattle, USA. AAAI Press.

Simons, P., Niemelä, I., \& Soininen, T. (2002). Extending and implementing the stable model semantics. Artifi cial Intelligence, 138, 181-234.

Son, T., \& Pontelli, E. (2006). A constructive semantic characterization of aggregates in anser set programming. Theory and Practice of Logic Programming. Accepted (available at http: / / arxiv.org/ abs/cs.AI/0601051).

Son, T., Pontelli, E., \& Tu, P. (2006). Answer sets for logic programs with arbitrary abstract constraint atoms. In Proceedings of the 21st National Conference on Artifi cial Intelligence (AAAI-06). AAAI Press.

Syrjänen, T. (1999). lparse, a procedure for grounding domain restricted logic programs. http://www . tcs.hut.fi/Software/smodels/lparse/.

Turner, H. (2003). Strong equivalence made easy: Nested expressions and weight constraints. Theory and Practice of Logic Programming, 3, (4\&5), 609-622.

Walser, J. (1997). Solving linear pseudo-boolean constraints with local search. In Proceedings of the 14th National Conference on Artifi cial Intelligence (AAAI-97), pp. 269-274. AAAI Press. 